\title{
COMPUTABLY REGULAR TOPOLOGICAL SPACES
}

\author{
KLAUS WEIHRAUCH
}

University of Hagen, Hagen, Germany

e-mail address: Klaus.Weihrauch@FernUni-Hagen.de

\begin{abstract}
This article continues the study of computable elementary topology started by the author and T. Grubba in 2009 and extends the author's 2010 study of axioms of computable separation. Several computable $T_{3^{-}}$and Tychonoff separation axioms are introduced and their logical relation is investigated. A number of implications between these axioms are proved and several implications are excluded by counter examples, however, many questions have not yet been answered. Known results on computable metrization of $T_{3}$-spaces from M. Schröder (1998) and T. Grubba, M. Schröder and the author (2007) are proved under uniform assumptions and with partly simpler proofs, in particular, the theorem that every computably regular computable topological space with non-empty base elements can be embedded into a computable metric space. Most of the computable separation axioms remain true for finite products of spaces.
\end{abstract}

\section{INTRODUCTION}

This article continues with the study of computable topology started in [13]. For computable topological spaces (as defined in [13]) in [12] we have introduced a number of computable versions of the topological $T_{0^{-}}, T_{1^{-}}$and $T_{2^{-}}$axioms and studied their relationship. In this article we define various computable versions of the topological $T_{3^{-}}$, Tychonoff- and $T_{4^{-}}$ axioms and compare them. Furthermore, we study computable metrization. For classical topology see, for example, [3]. In addition to new material we include earlier results from [7, [4, 5, 14] and [11] (in [1]) some of which have been proved under slightly differing assumptions and give some simpler proofs.

We will use the representation approach of computable analysis [6, 9, 2]. As the basic computability structure we start with computable topological spaces as introduced in [13. Notice that there are other slightly differing not equivalent definitions of "computable topological space" in other publications, in particular in [9]. We will use the notations and results from [13] some of which are mentioned very shortly in Section 2,

In Section 3 we introduce axioms for computable $T_{2}$ (2 axioms, which are alredy studied in [12]), for computable $T_{3}$ (3 axioms), for computable Tychonoff (3 axioms) and for computable $T_{4}$ and computable Urysohn. We give some examples and prove that the axioms do

2012 ACM CCS: [Mathematics of computing]: Continuous mathematics-topology-point set topology.

Key words and phrases: computable analysis, computable topology, axioms of separation. 
not depend on the details of the computable topological space but only on the computability concept defined by it.

In Section 4 we prove a number of implications between the introduced axioms.

In Section 5 we show by counterexamples that some implications are false. We summarize the results and list some open problems concerning the implications between the axioms. We also prove that computable $T_{3}$ and computable Tychonoff as well as their strong versions are equivalent for computable topological spaces with non-empty base elements.

In Section [6] we resume results on computable metrization from [7, 4, 5] and prove them under common weak assumptions. In particular we give a considerably simpler proof of the main theorem from [4] on the embedding computable $T_{3}$-spaces in computable metric spaces.

Each of the introduced computable separation classes is closed under the subspace operations, and most of them are closed under Cartesian product (Section 7).

\section{Preliminaries}

We will use the terminology and abbreviations summarized in [13, Section 2] and also results from [13. For further details see [9, 10, 2].

Let $\Sigma$ be a finite alphabet such that $0,1 \in \Sigma$. By $\Sigma^{*}$ we denote the set of finite words over $\Sigma$ and by $\Sigma^{\omega}$ the set of infinite sequences $p: \mathbb{N} \rightarrow \Sigma$ over $\Sigma, p=(p(0) p(1) \ldots)$. For a word $w \in \Sigma^{*}$ let $|w|$ be its length and let $\varepsilon \in \Sigma^{*}$ be the empty word. For $p \in \Sigma^{\omega}$ let $p^{<i} \in \Sigma^{*}$ be the prefix of $p$ of length $i \in \mathbb{N}$. We use the "wrapping function" $\iota: \Sigma^{*} \rightarrow \Sigma^{*}$, $\iota\left(a_{1} a_{2} \ldots a_{k}\right):=110 a_{1} 0 a_{2} 0 \ldots a_{k} 011$ for coding words such that $\iota(u)$ and $\iota(v)$ cannot overlap properly. Let $\langle i, j\rangle:=(i+j)(i+j+1) / 2+j$ be the bijective Cantor pairing function on $\mathbb{N}$. We consider standard functions for finite or countable tupling on $\Sigma^{*}$ and $\Sigma^{\omega}$ denoted by $\langle\cdot\rangle$ [9, Definition 2.1.7], in particular, $\left\langle u_{1}, \ldots, u_{n}\right\rangle=\iota\left(u_{1}\right) \ldots \iota\left(u_{n}\right),\langle u, p\rangle=\iota(u) p,\langle p, q\rangle=$ $(p(0) q(0) p(1) q(1) \ldots)$ and $\left\langle p_{0}, p_{1}, \ldots\right\rangle\langle i, j\rangle=p_{i}(j)$ for $u, u_{1}, u_{2}, \ldots \in \Sigma^{*}$ and $p, q, p_{0}, p_{1}, \ldots \in$ $\Sigma^{\omega}$. Consider $u \in \Sigma^{*}$ and $w \in \Sigma^{*} \cup \Sigma^{\omega}$. Let $u \sqsubseteq w$ iff $\iota(u)$ is a prefix of $w, u \ll w$ iff $\iota(u)$ is a subword of $w$ and let $\widehat{w}$ be the longest subword $v \in 11 \Sigma^{*} 11$ of $w$ (and the empty word if no such subword exists). Then for $u, w_{1}, w_{2} \in \Sigma^{*},\left(u \ll w_{1} \vee u \ll w_{2}\right) \Longleftrightarrow u \ll \widehat{w}_{1} \widehat{w}_{2}$.

For $Y_{0}, \ldots, Y_{n} \in\left\{\Sigma^{*}, \Sigma^{\omega}\right\}$ a partial function $f: \subseteq Y_{1} \times \ldots \times Y_{n} \rightarrow Y_{0}$ is computable, if it is computed by a Type-2 machine. A Type-2 machine $M$ is a Turing machine with $n$ input tapes, one output tape and finitely many additional work tapes. A specification assigns to the input tapes $1, \ldots, n$ and the output tape 0 types $Y_{i} \in\left\{\Sigma^{*}, \Sigma^{\omega}\right\}$ such that the machine computes a function $f_{M}: \subseteq Y_{1} \times \ldots \times Y_{n} \rightarrow Y_{0}[9]$. Notice that on the output tape the machine can only write and move its head to the right.

A notation of a set $X$ is a surjective partial function $\nu: \subseteq \Sigma^{*} \rightarrow X$ and a representation is a surjective partial function $\delta: \subseteq \Sigma^{\omega} \rightarrow X$. Here, finite or infinite sequences of symbols are considered as "concrete names" of the "abstract" elements of $X$. Computability on $X$ is defined by computations on names. Let $\gamma_{i}: \subseteq Y_{i} \rightarrow X_{i}, Y_{i} \in\left\{\Sigma^{*}, \Sigma^{\omega}\right\}$ for $i \in\{0,1\}$ be notations or representations. A set $W \subseteq X_{0}$ is called $\gamma_{0}$-r.e. (recursively enumerable), if there is a Type-2 machine $M$ that halts on input $y_{0} \in \operatorname{dom}\left(\gamma_{0}\right)$ iff $\gamma_{0}\left(y_{0}\right) \in W$. A function $h: \subseteq Y_{1} \rightarrow Y_{0}$ realizes a multi-function $f: X_{1} \rightrightarrows X_{0}$, iff $\gamma_{0} \circ h\left(y_{1}\right) \in f \circ \gamma_{1}\left(y_{1}\right)$ whenever $\left.f \circ \gamma_{1}\left(y_{1}\right)\right) \neq \emptyset$. The function $f$ is called $\left(\gamma_{1}, \gamma_{0}\right)$-computable, if it has a computable realization. The definitions can be generalized straightforwardly to subsets of $X_{1} \times \ldots \times X_{n}$ and multi-functions $f: X_{1} \times \ldots \times X_{n} \rightarrow X_{0}\left(\left(\gamma_{1}, \ldots, \gamma_{n}\right)\right.$-r.e., $\left(\gamma_{1}, \ldots, \gamma_{n}, \gamma_{0}\right)$-computable $)$. 
In this article we study axioms of computable separation for computable topological spaces $\mathbf{X}=(X, \tau, \beta, \nu)$ [13, Definition 4], where $\tau$ is a $T_{0}$-topology on the set $X$ and $\nu: \subseteq \Sigma^{*} \rightarrow \beta$ is a notation of a base $\beta$ of $\tau$ such that $\operatorname{dom}(\nu)$ is recursive and there is an r.e. set $S \subseteq(\operatorname{dom}(\nu))^{3}$ such that $\nu(u) \cap \nu(v)=\bigcup\{\nu(w) \mid(u, v, w) \in S\}$. We mention expressly that in the past various spaces have been called "computable topological space". We allow $U=\emptyset$ for $U \in \beta$ which is forbidden, for example, in [5, 14].

We define a notation $\nu^{\mathrm{fs}}$ of the finite subsets of the base $\beta$ by $\nu^{\mathrm{fs}}(w)=W: \Longleftrightarrow((\forall v \ll$ $w) v \in \operatorname{dom}(\nu) \wedge W=\{\nu(v) \mid v \ll w\})$. Then $\bigcup \nu^{\mathrm{fs}}$ and $\bigcap \nu^{\mathrm{fs}}$ are notations of the finite unions and the finite intersections of base elements, respectively.

For the points of $X$ we consider the canonical (or inner) representation $\delta: \subseteq \Sigma^{\omega} \rightarrow X$; $\delta(p)=x$ iff $p$ is a list of all $\iota(u)$ (possibly padded with $1 \mathrm{~s}$ ) such that $x \in \nu(u)$ (hence $u \ll p \Longleftrightarrow \delta(p) \in \nu(u))$. For the set of open sets, the topology $\tau$ we consider the inner representation $\theta: \subseteq \Sigma^{\omega} \rightarrow \tau$ defined by $u \in \operatorname{dom}(\nu)$ if $u \ll p \in \operatorname{dom}(\theta)$ and $\theta(p):=\bigcup\{\nu(u) \mid$ $u \ll p\}$. For the closed sets we consider the outer representation $\psi^{-}(p):=X \backslash \theta(p)$ [13].

The canonical notations of the natural and the rational numbers are denoted by $\nu_{\mathbb{N}}$ and $\nu_{\mathbb{Q}}$, respectively. For the real numbers we use the canonical representation $\rho$ (Example 3.3(1) $)$, the lower representation $\rho_{<}$and the upper representation $\rho_{>}[9]$.

\section{Axioms of Computable separation}

For a topological space $\mathbf{X}=(X, \tau)$ with set $\mathcal{A}$ of closed sets we consider the following separation properties:

Definition 3.1 (axioms of separation).

$$
\begin{array}{ll}
\mathrm{T}_{0}: & (\forall x, y \in X, x \neq y)(\exists W \in \tau)((x \in W \wedge y \notin W) \vee(x \notin W \wedge y \in W))), \\
\mathrm{T}_{1}: & (\forall x, y \in X, x \neq y)(\exists W \in \tau)(x \in W \wedge y \notin W), \\
\mathrm{T}_{2}: & (\forall x, y \in X, x \neq y)(\exists U, V \in \tau)(U \cap V=\emptyset \wedge x \in U \wedge y \in V), \\
\mathrm{T}_{3}: & (\forall x \in X, \forall A \in \mathcal{A}, x \notin A)(\exists U, V \in \tau)(U \cap V=\emptyset \wedge x \in U \wedge A \subseteq V), \\
\mathrm{Ty}: & (\forall x \in X, \forall A \in \mathcal{A}, x \notin A)(\exists f: X \rightarrow \mathbb{R}) \\
& (f \text { is continuous, range }(f) \subseteq[0 ; 1], f(x)=0 \text { and } f[A] \subseteq\{1\}) \\
\mathrm{T}_{4}: & (\forall A, B \in \mathcal{A}, A \cap B=\emptyset)(\exists U, V \in \tau)(U \cap V=\emptyset \wedge A \subseteq U \wedge B \subseteq V) . \\
\mathrm{Ur}: & (\forall A, B \in \mathcal{A}, A \cap B=\emptyset)(\exists f: X \rightarrow \mathbb{R}) \\
& (f \text { is continuous, range }(f) \subseteq[0 ; 1], f[A] \subseteq\{0\} \text { and } f[B] \subseteq\{1\} .)
\end{array}
$$

We will speak of $T_{2}$-spaces, $T y$-spaces etc.

$T_{2}$-spaces are called Hausdorff spaces. Many authors, for example [3], call a space $T_{3^{-}}$ space or regular iff $T_{1}+T_{3}$, call a space $T_{3 \frac{1}{2}}$-space, Tychonoff space or completely regular iff $\mathrm{T}_{1}+\mathrm{Ty}$, and call a space $T_{4}$-space or normal iff $\mathrm{T}_{1}+\mathrm{T}_{4}$. From topology [3] we know:

$$
\mathrm{T}_{1}+\mathrm{Ur} \Longleftrightarrow \mathrm{T}_{1}+\mathrm{T}_{4} \Longrightarrow \mathrm{T}_{1}+\mathrm{Ty} \Longrightarrow \mathrm{T}_{1}+\mathrm{T}_{3} \Longrightarrow \mathrm{T}_{2} \Longrightarrow \mathrm{T}_{1} \Longrightarrow \mathrm{T}_{0}
$$

where the implications are proper. The first implication from the right to the left is Urysohn's lemma. We mention that $(X, \tau)$ is a $T_{1}$-space, iff all sets $\{x\}(x \in X)$ are closed [3]. 
In this article we consider only computable topological spaces $\mathbf{X}=(X, \tau, \beta, \nu)$, which are $T_{0}$-spaces with countable base (also called second countable). For such spaces $\mathrm{T}_{3} \Longrightarrow \mathrm{T}_{2}$ and $\mathrm{T}_{1}+\mathrm{T}_{4} \Longleftrightarrow \mathrm{T}_{1}+\mathrm{T}_{3}[3$, Theorem 1.5.16], hence

$$
\mathrm{T}_{1}+\mathrm{Ur} \Longleftrightarrow \mathrm{T}_{1}+\mathrm{T}_{4} \Longleftrightarrow \mathrm{T}_{1}+\mathrm{Ty} \Longleftrightarrow \mathrm{T}_{3} .
$$

Axioms of computable separation for $T_{0}, T_{1}$ and $T_{2}$ have been studied in [12]. In the following we introduce computable versions of the axioms $\mathrm{T}_{3}, \mathrm{Ty}, \mathrm{T}_{4}$ and Ur. The computable Hausdorff axioms $\mathrm{CT}_{2}$ and $\mathrm{SCT}_{2}$ are from [12]. In the direct effectivizations the existing objects must be computed. For the points we compute basic neighborhoods (w.l.o.g.) instead of general open neighborhoods. Let $C(X, \mathbb{R})$ be the set of continuous functions $f: X \rightarrow \mathbb{R}$ and let $[\delta \rightarrow \rho]$ be the canonical representation of this set [13, 9].

Definition 3.2 (axioms of computable separation).

$\mathrm{CT}_{2}$ : The multi-function $t_{2}: X \times X \rightrightarrows \beta \times \beta$ is $(\delta, \delta,[\nu, \nu])$-computable where $(U, V) \in t_{2}(x, y)$ iff $x \in U, y \in V$ and $U \cap V=\emptyset$.

$\mathrm{SCT}_{2}:$ There is an r.e. set $H \subseteq \Sigma^{*} \times \Sigma^{*}$ such that

$$
\begin{aligned}
& (\forall x, y, x \neq y)(\exists(u, v) \in H)(x \in \nu(u) \wedge y \in \nu(v)) \quad \text { and } \\
& (\forall(u, v) \in H) \nu(u) \cap \nu(v)=\emptyset .
\end{aligned}
$$

$\mathrm{WCT}_{3}$ : The multi-function $t_{3}^{w}: X \times \beta \rightrightarrows \beta$ is $(\delta, \nu, \nu)$-computable where $U \in t_{3}^{w}(x, W)$ iff $x \in U \subseteq \bar{U} \subseteq W$.

$\mathrm{CT}_{3}$ : The multi-function $t_{3}: X \times \mathcal{A} \rightrightarrows \beta \times \tau$ is $\left(\delta, \psi^{-},[\nu, \theta]\right)$-computable, where $(U, V) \in t_{3}(x, A)$ iff $x \notin A, U \cap V=\emptyset, x \in U$ and $A \subseteq V$.

$\mathrm{CT}_{3}^{\prime}$ : The multi-function $t_{3}^{\prime}: X \times \beta \rightrightarrows \beta \times \mathcal{A}$ is $\left(\delta, \nu,\left[\nu, \psi^{-}\right]\right)$-computable where $(U, B) \in t_{3}^{\prime}(x, W)$ iff $x \in U \subseteq B \subseteq W$.

$\mathrm{SCT}_{3}$ : There are an r.e. set $R \subseteq \operatorname{dom}(\nu) \times \operatorname{dom}(\nu)$ and a computable function $r: \subseteq \Sigma^{*} \times \Sigma^{*} \rightarrow \Sigma^{\omega}$ such that for all $u, w \in \operatorname{dom}(\nu)$,

$$
\begin{aligned}
& \nu(w)=\bigcup\{\nu(u) \mid(u, w) \in R\}, \\
& (u, w) \in R \Longrightarrow \nu(u) \subseteq \psi^{-} \circ r(u, w) \subseteq \nu(w) .
\end{aligned}
$$

CTy : The multi-function $t_{\mathrm{Ty}}: X \times \mathcal{A} \rightrightarrows C(X, \mathbb{R})$ is $\left(\delta, \psi^{-},[\delta \rightarrow \rho]\right)$-computable where $f \in t_{\mathrm{Ty}}(x, A)$ iff range $(f) \subseteq[0 ; 1], x \notin A, f(x)=0$ and $f[A] \subseteq\{1\}$.

$\mathrm{CTy}^{\prime}$ : The multi-function $t_{\mathrm{Ty}}^{\prime}: X \times \beta \rightrightarrows \beta \times C(X, \mathbb{R})$ is $(\delta, \nu,[\nu,[\delta \rightarrow \rho]])$-computable where $(U, f) \in t_{\mathrm{Ty}}^{\prime}(x, W)$ iff range $(f) \subseteq[0 ; 1], x \in U \subseteq W, f[U]=\{0\}$ and $f[X \backslash W] \subseteq\{1\}$.

SCTy : There are an r.e. set $T \subseteq \operatorname{dom}(\nu) \times \operatorname{dom}(\nu)$ and a computable function $t: \subseteq \Sigma^{*} \times \Sigma^{*} \rightarrow \Sigma^{\omega}$ such that

$$
\begin{gathered}
\nu(w)=\bigcup\{\nu(u) \mid(u, w) \in T\} \quad \text { for all } w \in \operatorname{dom}(\nu) \text { and } \\
f_{u w}[\nu(u)] \subseteq\{0\} \text { and } f_{u w}[X \backslash \nu(w)] \subseteq\{1\} \quad \text { for all }(u, w) \in T,
\end{gathered}
$$

where $f_{u w}:=[\delta \rightarrow \rho] \circ t(u, w)$.

$\mathrm{CT}_{4}$ : The multi-function $t_{4}: \mathcal{A} \times A \rightrightarrows \tau \times \tau$ is $\left(\psi^{-}, \psi^{-},[\theta, \theta]\right)$-computable where $(U, V) \in t_{4}(A, B)$ iff $U \cap V=\emptyset, A \subseteq U$ and $B \subseteq V$.

CUr : The multi-function $t_{\mathrm{Ur}}: \mathcal{A} \times A \rightrightarrows C(X, \mathbb{R})$ is $\left(\psi^{-}, \psi^{-},[\delta \rightarrow \rho]\right)$-computable, where $f \in t_{\mathrm{Ur}}(A, B)$ iff range $(f) \subseteq[0 ; 1], A \cap B=\emptyset, f[A] \subseteq\{0\}$ and $f[B] \subseteq\{1\}$. 
The axioms $\mathrm{CT}_{2}, \mathrm{CT}_{3}, \mathrm{CTy}, \mathrm{CT}_{4}$ and $\mathrm{CUr}$ are the direct effectivizations of $T_{2}, \mathrm{~T}_{3}$, Ty, $\mathrm{T}_{4}$ and Ur, respectively. Obviously, $\mathrm{SCT}_{2}$ implies $\mathrm{T}_{2} . \mathrm{WCT}_{3}, \mathrm{CT}_{3}, \mathrm{CT}_{3}^{\prime}$ and $\mathrm{SCT}_{3}$ imply $\mathrm{T}_{3}$. CTy, $\mathrm{CTy}^{\prime}$ and SCTy imply Ty. $\mathrm{CT}_{4}$ implies $\mathrm{T}_{4}$. CUr implies Ur. In contrast to $\mathrm{CT}_{3}^{\prime}$, in $\mathrm{WCT}_{3}$ the function $t_{3}^{w}$ does not compute a $\psi^{-}$-name of a closed set such that $x \in U \subseteq B \subseteq W$. The sets $H$ from $\mathrm{SCT}_{2}, R$ from $\mathrm{SCT}_{3}$ and $T$ from $\mathrm{SCTy}$ may contain pairs $(u, w)$ such that $\nu(u)=\emptyset$ or $\nu(w)=\emptyset$. Also, empty open or closed sets are not excluded as inputs for the separating functions.

We do not consider the numerous variants of the separation axioms where in some places the representations $\delta$ of the points, $\theta$ of the open sets and $\psi^{-}$of the closed sets are replaced by $\delta^{-}, \theta^{-}$and $\psi^{+}$, respectively [13, Definition 5]. The following examples illustrate the definitions. Further examples are given in Section 5 .

\section{Example 3.3.}

(1) The computable real line is defined by $\mathbf{R}:=\left(\mathbb{R}, \tau_{\mathbb{R}}, \beta, \nu\right)$ such that $\tau_{\mathbb{R}}$ is the real line topology and $\nu$ is a canonical notation of the set of all open intervals with rational endpoints. $\mathbf{R}$ is a computable topological space. Its canonical representation is called $\rho$. All the axioms from Definition 3.2 are true for $\mathbf{R}$.

(2) A computable metric space is a tuple $\mathbf{M}=(X, d, A, \alpha)$ such that $(M, d)$ is a metric space and $\alpha$ is a notation with recursive domain of a set $A$ which is dense in $X$ such that the distance $\mathrm{d}$ restricted to $M \times M$ is $(\alpha, \alpha, \rho)$-computable [9, Definition 8.1.2]. Let $\nu$ be a canonical notation of the set $\beta$ of all open balls with center from $A$ and rational radius and let $\tau$ be the smallest topology containing $\beta$. Then $\mathbf{X}=(X, \tau, \beta, \nu)$ is a computable topological space for which all the axioms from Definition 3.2 are true (Theorem 6.2).

(3) ( $C T_{0}$ and $C T_{4}$ but not $T_{1}, T_{2}$ or $\left.T_{3}\right)$ A space is $C T_{0}$ iff the multi-function $t_{0}$ is $(\delta, \delta, \nu)$ computable, where $t_{0}$ maps every $(x, y) \in X^{2}$ such that $x \neq y$ to some $U \in \beta$ such that $(x \in U$ and $y \notin U)$ or $(x \notin U$ and $y \in U)$ [12].

Let $\mathbf{S i}:=\left(\{\perp, \top\}, \tau_{\mathbf{S i}}, \beta_{\mathbf{S i}}, \nu_{\mathbf{S i}}\right)$ be the Sierpinski space defined by $\nu_{\mathbf{S i}}(0)=\{\perp, \top\}$ and $\nu_{\mathbf{S i}}(1)=\{\top\}$. The space is $T_{0}$ but not $T_{1}$.

There is a machine $M$ that on input $(p, q) \in \Sigma^{\omega} \times \Sigma^{\omega}$ writes 1 and halts. The function $f_{M}$ realizes the function $(x, y) \mapsto\{\top\}$. Then for $x \neq y,(x=\top$ and $y=\perp)$ or $(x=\perp$ and $y=\top)$, hence for $U:=\{\top\}=\nu(1),(x \in U$ and $y \notin U)$ or $(x \notin U$ and $y \in U)$. Therefore, $\mathbf{S i}$ is $C T_{0}$.

There are computable sequences $p^{\prime}, q^{\prime} \in \Sigma^{\omega}$ such that $\theta_{\mathbf{S i}}\left(p^{\prime}\right)=\emptyset$ and $\theta_{\mathbf{S i}}\left(q^{\prime}\right)=$ $\{\perp, \top\}$. There is a machine $M$ that on input $(p, q)$ searches in $p$ and $q$ until it has found $0 \ll p$ or $0 \ll q$. In the first case it writes $\left\langle p^{\prime}, q^{\prime}\right\rangle$ and in the second case $\left\langle q^{\prime}, p^{\prime}\right\rangle$. Let $\psi_{\mathbf{S i}}^{-}(p)=A$ and $\psi_{\mathbf{S i}}^{-}(q)=B$ such that $A \cap B=\emptyset$. Then $A=\emptyset$ or $B=\emptyset$, hence $0 \ll p$ or $0 \ll q$. In the first case, $A=\emptyset \subseteq \theta_{\mathbf{S i}}\left(p^{\prime}\right)$ and $B \subseteq \theta_{\mathbf{S i}}\left(q^{\prime}\right)=\{\perp, \top\}$ and in the second case, $A \subseteq \theta_{\mathbf{S i}}\left(q^{\prime}\right)=\{\perp, \top\}$ and $B=\emptyset \subseteq \theta_{\mathbf{S i}}\left(p^{\prime}\right)$. Therefore, $f_{M}$ realizes $t_{4}$.

(4) (discrete implies $W C T_{3}$ ) Let $\mathbf{X}$ be a discrete computable topological space. Then every subset of $X$ is open and closed and $X$ is countable. The function $t_{3}^{w}:(x, W) \mapsto W$ is $(\delta, \nu, \nu)$-computable. It satisfies $\mathrm{WCT}_{3}$ since $x \in W$ implies $x \in \bar{W} \subseteq W$.

By the next lemma the above computable separation axioms are robust, that is, they do not depend on the notation $\nu$ of the base explicitly but only on the computability concept on the points induced by it. Two computable topological spaces $\mathbf{X}=(X, \tau, \beta, \nu)$ and $\widetilde{\mathbf{X}}=(\widetilde{X}, \widetilde{\tau}, \widetilde{\beta}, \widetilde{\nu})$ are called equivalent iff $(X, \tau)=(\widetilde{X}, \widetilde{\tau}), \nu \leq \widetilde{\theta}$ and $\widetilde{\nu} \leq \theta$, that is, there are 
computable functions $g, \widetilde{g}: \subseteq \Sigma^{*} \rightarrow \Sigma^{\omega}$ such that

$$
\nu(u)=\widetilde{\theta} \circ g(u) \text { and } \widetilde{\nu}(u)=\theta \circ \widetilde{g}(u) .
$$

The condition " $\nu \leq \widetilde{\theta}$ and $\widetilde{\nu} \leq \theta$ " is equivalent to $\delta \equiv \widetilde{\delta}$. For equivalent topological spaces, $\theta \equiv \widetilde{\theta}, \psi^{-} \equiv \widetilde{\psi}^{-}$and $\kappa \equiv \widetilde{\kappa}[13$, Definition 21, Theorem 22].

Lemma 3.4. Let $\widetilde{\mathbf{X}}=(X, \tau, \widetilde{\beta}, \widetilde{\nu})$ be a computable topological space equivalent to $\mathbf{X}=$ $(X, \tau, \beta, \nu)$. Then each separation axiom from Definition 3.2 for $\mathbf{X}$ is equivalent to the corresponding axiom for $\widetilde{\mathbf{X}}$.

\section{Proof:}

$\mathrm{SCT}_{2}$ : See [12].

$\mathbf{W C T}_{3}$ : Assume $\widetilde{\mathrm{WCT}}_{3}$. Let $x=\delta(p), W=\nu(w)$ and $x \in W$. Since $\delta \equiv \widetilde{\delta}$ and $\nu \leq \widetilde{\theta}$ we can compute some $\widetilde{p}$ and some $\widetilde{w}$ such that $x=\widetilde{\delta}(\widetilde{p}) \in \widetilde{\nu}(\widetilde{w}) \subseteq \nu(w)$. By ${\widetilde{\mathrm{WCT}_{3}}}_{3}$ we can compute some $\widetilde{u}$ such that $x \in \widetilde{\nu}(\widetilde{u}) \subseteq \operatorname{closure}(\widetilde{\nu}(\widetilde{u})) \subseteq \widetilde{\nu}(\widetilde{w})$. Since $\widetilde{\nu} \leq \theta$, from $p$ and $\widetilde{u}$ we can compute some $u$ such that $x \in \nu(u) \subseteq \nu(\widetilde{u})$. We obtain $x \in \nu(u) \subseteq \overline{\nu(u)} \subseteq \nu(w)$. Therefore, $\mathrm{WCT}_{3}$ is true. By symmetry, $\mathrm{WCT}_{3} \Longrightarrow{\widetilde{\mathrm{WCT}_{3}}}_{3}$.

$\mathbf{S C T}_{3}$ : Assume $\mathrm{SCT}_{3}$. With the functions $g, \widetilde{g}$ from (3.8) let $\widetilde{R}:=\{(\widetilde{u}, \widetilde{w}) \mid(\exists(u, w) \in$ $R)(w \ll \widetilde{g}(\widetilde{w}), \widetilde{u} \ll g(u))\}$. Then $\widetilde{R}$ is r.e. Suppose $(\widetilde{u}, \widetilde{w}) \in \widetilde{R}$. Then for some $(u, w) \in R$, $\widetilde{\nu}(\widetilde{u}) \subseteq \widetilde{\theta} \circ g(u)=\nu(u) \subseteq \nu(w) \subseteq \theta \circ \widetilde{g}(\widetilde{w})=\widetilde{\nu}(\widetilde{w})$. On the other hand suppose, $x \in \widetilde{\nu}(\widetilde{w})=$ $\theta \circ \widetilde{g}(\widetilde{w})$. Then $x \in \nu(w)$ for some $w \ll \widetilde{g}(\widetilde{w})$. By $\mathrm{SCT}_{3}$ there is some $u$ such that $(u, w) \in R$ and $x \in \nu(u)=\widetilde{\theta} \circ g(u)$. Then $x \in \widetilde{\nu}(\widetilde{u})$ for some $\widetilde{u} \ll g(u)$. In summary, $x \in \widetilde{\nu}(\widetilde{u})$ for some $\widetilde{u}$ such that $(\widetilde{u}, \widetilde{w}) \in \widetilde{R}$. Therefore, (3.4) holds for $\widetilde{\nu}$ and $\widetilde{R}$.

There is a computable function $d$ translating $\psi^{-}$to $\widetilde{\psi}^{-}$[13]. Let $M$ be a machine that on input $(\widetilde{u}, \widetilde{w})$ searches for $(u, w) \in R$ such that $w \ll \widetilde{g}(\widetilde{w})$ and $\widetilde{u} \ll g(u)$ and then computes $d \circ r(u, w)$. Then $\widetilde{\nu}(\widetilde{u}) \subseteq \widetilde{\theta} \circ g(u)=\nu(u) \subseteq \psi^{-} \circ r(u, w) \subseteq \nu(w) \subseteq \theta \circ \widetilde{g}(\widetilde{w})=\widetilde{\nu}(\widetilde{w})$. Since $\psi^{-} \circ r(u, w)=\widetilde{\psi}^{-} \circ d \circ r(u, w)=\widetilde{\psi}^{-} \circ f_{M}(\widetilde{u}, \widetilde{w})$, (3.5) holds for $\widetilde{S C T}_{3}$ with $\widetilde{r}:=f_{M}$ and $\widetilde{R}$. Therefore, $\mathrm{SCT}_{3} \Longrightarrow{\widetilde{\mathrm{SCT}_{3}}}_{3}$. By symmetry, $\widetilde{\mathrm{SCT}}_{3} \Longrightarrow \mathrm{SCT}_{3}$.

For the other axioms the proofs are similar. Notice that $[\delta \rightarrow \rho] \equiv[\widetilde{\delta} \rightarrow \rho]$ if $\delta \equiv \widetilde{\delta}$.

\section{IMPLiCATIONS}

In this section we prove a number of implications between the separation properties, in Section 5 we prove by counterexamples that some of the implications are proper. A topological space is discrete iff every singleton $\{x\}$ is open iff every subset $B \subseteq X$ is open. A discrete space is $T_{i}$ for $i=1, \ldots, 4$. Let $\mathrm{D}$ be the axiom stating that the space is discrete.

\section{Theorem 4.1.}

(1) $\mathrm{SCT}_{3} \Longrightarrow \mathrm{CTy} \Longrightarrow \mathrm{CT}_{3} \Longrightarrow \mathrm{SCT}_{2} \Longrightarrow \mathrm{CT}_{2}$,

(2) $\mathrm{D} \Longrightarrow \mathrm{WCT}_{3}$,

(3) $\mathrm{CT}_{3} \Longrightarrow \mathrm{WCT}_{3}$

(4) $\mathrm{SCT}_{3} \Longrightarrow \mathrm{CT}_{4}$,

(5) $\mathrm{SCT}_{3} \Longleftrightarrow \mathrm{SCTy}, \mathrm{CTy} \Longleftrightarrow \mathrm{CTy}^{\prime}, \mathrm{CT}_{3} \Longleftrightarrow \mathrm{CT}_{3}^{\prime}$,

(6) $\mathrm{CT}_{4} \Longleftrightarrow \mathrm{CUr}$. 
The implications $\mathrm{SCT}_{3} \Longrightarrow \mathrm{CT}_{4} \Longrightarrow \mathrm{CUr}$ have been already been proved in [7] for a computable topological space $T(\mathbf{Z})$ derived from a predicate space $\mathbf{Z}$ (in the terminology of [13]). For our computable topological space $\mathbf{X}=(X, \tau, \beta, \nu), \mathbf{Z}:=(X, \beta, \nu)$ is a predicate space and $T(\mathbf{Z})=(X, \tau, \tilde{\beta}, \tilde{\nu})$, where $\tilde{\nu}$ is the notation of the finite intersections of base elements canonically derived from $\nu$, is equivalent to $\mathbf{X}$ by [13, Lemma 23]. By Lemma 3.4. $\mathrm{SCT}_{3} \Longrightarrow \mathrm{CT}_{4} \Longrightarrow \mathrm{CUr}$ for a computable topological space follows from [7. More concise proofs are given in [4] for a computable topological space $\mathbf{X}=(X, \tau, \beta, \nu)$ such that $U \neq \emptyset$ for all $U \in \beta$. This restriction, however, is unnecessary. The reader may check this in Appendix A.

\section{Proof:}

$\mathbf{S C T}_{\mathbf{3}} \Longrightarrow \mathbf{C T}_{4}$ : (cf. [3, Lemma 1.5.15, Theorem 1.5.17]) The proof from [4] is added in Appendix B.

$\mathbf{C T}_{\mathbf{4}} \Longrightarrow \mathbf{C U r}$ : See [3, Theorem 1.5.15]. The proof from [4] is added in the appendix.

$\mathbf{C U r} \Longrightarrow \mathbf{C T}_{4}$ : By the multi-function $t_{\mathrm{UR}}$ from $A, B$ such that $A \cap B=\emptyset$ we can compute a continuous function $f: X \rightarrow \mathbb{R}$ such that range $(f) \subseteq[0 ; 1], f[A] \subseteq\{0\}$ and $f[B] \subseteq\{1\}$. Then by [13, Theorem 38] the open sets $U:=f^{-1}[(-\infty ; 1 / 2)]$ and $V:=$ $\left.f^{-1}[(1 / 2) ; \infty)\right]$ can be computed. They separate $A$ and $B$.

$\mathbf{C U r}+\mathbf{S C T}_{\mathbf{3}} \Longrightarrow \mathbf{S C T y}$ : Let $R$ be the set and let $r$ be the function from $\mathrm{SCT}_{3}$. Define $T:=R$. By $r$ from $(u, w) \in T$ we can compute a closed set $A$ such that $\nu(u) \subseteq A \subseteq \nu(w)$. Since $U \mapsto U^{c}$ for base sets is $\left(\nu, \psi^{-}\right)$-computable, by $t_{U r}$ from $A$ and $X \backslash \nu(w)$ we can compute some continuous function $f: X \rightarrow \mathbb{R}$ such that range $(f) \subseteq[0 ; 1], f[A] \subseteq\{0\}$, hence $f[\nu(u)] \subseteq\{0\}$ and $f[X \backslash \nu(w)] \subseteq\{1\}$.

SCTy $\Longrightarrow \mathbf{S C T}_{\mathbf{3}}$ : Let $T$ be the set and $t$ be the function from SCTy. Define $R:=T$. By [13, Theorem 38] the function $f \mapsto f^{-1}(1 / 2, \infty)$ for continuous $f: X \rightarrow \mathbb{R}$ is $([\delta \rightarrow \rho], \theta)$ computable. Let $h: \subseteq \Sigma^{\omega} \rightarrow \Sigma^{\omega}$ be a computable realization. Then for $(u, w) \in R$ and $V:=([\delta \rightarrow \rho] \circ t(u, w))^{-1}(1 / 2 ; \infty)=\theta \circ h \circ t(u, w), \nu(u) \cap V=\emptyset$ and $X \backslash \nu(w) \subseteq V$. Therefore, $\nu(u) \subseteq X \backslash V=\psi^{-} \circ h \circ t(u, w)=X \backslash V \subseteq \nu(w)$. Define $r:=h \circ t$.

$\mathbf{C T y}^{\prime} \Longrightarrow \mathbf{C T y}$ : From $(x, A)$ such that $x \notin A$ some $W \in \beta$ can be computed such that $x \in W \subseteq X \backslash A$. From $(x, W)$ such that $x \in W$ by $t_{\mathrm{Ty}}^{\prime}$ some $U \in \beta$ and some continuous function $f: X \rightarrow \mathbb{R}$ can be computed such that $x \in U \subseteq W$, range $(f) \subseteq[0 ; 1], f(y)=0$ for $y \in U$ and $f(y)=1$ for $y \notin W$. For this function $f, f(x)=0$ since $x \in U$ and $f(y)=1$ for $y \in A$ since $W \subseteq X \backslash A$. Therefore, $t_{\mathrm{Ty}}$ is $\left(\delta, \psi^{-},[\delta \rightarrow \rho]\right)$-computable.

$\mathbf{C T y} \Longrightarrow \mathbf{C T y}^{\prime}$ : Suppose $x \in W \in \beta$. From $W, A:=X \backslash W$ can be computed. From $(x, A)$ by $t_{\mathrm{Ty}}$ some continuous function $g$ can be computed such that range $(g) \subseteq[0 ; 1]$, $g(x)=0$ and $g(y)=1$ for $y \in A$. Let $f(y):=\max (0,2 g(y)-1)$. Then $g \mapsto f$ is $([\delta \rightarrow$ $\rho],[\delta \rightarrow \rho])$-computable. Obviously range $(f) \subseteq[0 ; 1], f(y)=0$ for $g(y)<1 / 2$ and $f(y)=1$ for $y \in A$. By [13, Theorem 38], $g \mapsto g^{-1}(-\infty, 1 / 2)$ is $([\delta \rightarrow \rho], \theta)$-computable. Finally from $(x, V)$ such that $x \in V \in \tau$ some $U \in \beta$ can be computed such that $x \in U \subseteq V$. Notice that $f(y)=0$ for $y \in U \subseteq g^{-1}(-\infty, 1 / 2)$. Therefore, from $(x, W)$ some $(U, f) \in t_{\mathrm{Ty}}(x, W)$ can be computed.

$\mathbf{C T}_{\mathbf{3}}^{\prime} \Longrightarrow \mathbf{C T}_{\mathbf{3}}$ : Using $t_{3}^{\prime}$ from $(x, A)$ we can compute in turn $(x, X \backslash A),(x, W)$ for some $W \in \beta$ such that $x \in W \subseteq X \backslash A$, some $(U, B) \in \beta \times \mathcal{A}$ such that $x \in U \subseteq B \subseteq W$, and finally $(U, V)$ where $V:=X \backslash B$. By simple transformations, $U \cap V=\emptyset, x \in U$ and $A \subseteq V$. 
$\mathbf{C T}_{\mathbf{3}} \Longrightarrow \mathbf{C T}_{\mathbf{3}}^{\prime}$ : The function $W \mapsto X \backslash W$ is $\left(\nu, \psi^{-}\right)$-computable, and the function $V \mapsto X \backslash V$ is $\left(\theta, \psi^{-}\right)$-computable. Then using $t_{3}$, from $(x, W)$ we can compute in turn $(x, A), A:=X \backslash W,(U, V) \in \beta \times \tau$ such that $U \cap V=\emptyset, x \in U$ and $A \subseteq V$, and $(U, B)$, $B:=X \backslash V$. By simple transformations, $x \in U \subseteq B \subseteq W$.

$\mathrm{CT}_{3}^{\prime} \Longrightarrow \mathrm{WCT}_{3}$ : Obvious.

SCTy $\Longrightarrow \mathbf{C T y}^{\prime}$ : Let $T$ be the set and $t$ be the function from SCTy. Assume $x=$ $\delta(p) \in \nu(w)$. By (3.6) there is some $u \in \operatorname{dom}(\nu)$ such that $u \ll p$, and $(u, w) \in T$. Then $f_{u w}:=[\delta \rightarrow \rho] \circ t(u, w)$ satisfies (3.7). There is a machine that on input $(p, w)$ searches for some $u$ such that $u \ll p$ and $(u, w) \in T$ and writes $\langle u, t(u, w)\rangle$. Then $f_{M}$ realizes $t_{\mathrm{Ty}}^{\prime}$.

CTy $\Longrightarrow \mathbf{C T}_{\mathbf{3}}$ : Suppose $x \notin A$ and $A$ is closed. By $t_{\mathrm{Ty}}$ we can compute some continuous function $f$ such that $f(x)=0$ and $f(y)=1$ for $x \in A$. By [13, Theorem 38], the functions $f \mapsto f^{-1}(-\infty, 1 / 2)$ and $f \mapsto f^{-1}(1 / 2, \infty)$ are $([\delta \rightarrow \rho], \theta)$-computable. Since $x \subseteq f^{-1}(-\infty, 1 / 2)$ and $A \subseteq f^{-1}(1 / 2, \infty)$, the multi-function $(x, A) \rightrightarrows(U, V)$ such that $x \in U, A \subseteq V$ and $U \cap V=\emptyset$ is $\left(\delta, \psi^{-},[\theta, \theta]\right)$-computable. From $q$ and $r$ such that $\delta(q) \in \theta(r)$ we can compute some $u$ such that $x \in \nu(u) \subseteq \theta(r)$. Therefore, $t_{3}$ is $\left(\delta, \psi^{-},[\nu, \theta]\right)$ computable.

$\mathbf{C T}_{3}^{\prime} \Longrightarrow \mathbf{S C T}_{2}$ : Let $M$ be a machine such that $f_{M}$ realizes $t_{3}^{\prime}$. Since finite intersection is $\left(\nu^{\mathrm{fs}}, \theta\right)$-computable [13, Theorem 11], there is a computable function $g$ such that $\bigcap \nu^{\mathrm{fs}}(w)=\theta \circ g(w)$. Let $H$ be the set of all $(u, v) \in \operatorname{dom}(\nu) \times \operatorname{dom}(\nu)$ with the following properties: there are words $w, u_{1}, v_{1}, v_{2}$ such that $v_{1} \in \operatorname{dom}\left(\nu^{\mathrm{fs}}\right), w \ll v_{1}, u \ll g\left(v_{1}\right)$ and on input $\left(v_{1} 1^{\omega}, w\right)$ in length $\left(v_{1}\right)$ steps the machine $M$ writes at least $\iota\left(u_{1}\right) v_{2}$ such that $u_{1} \ll v_{1}$, and $v \ll v_{2}$. The set $H$ is r.e.

Suppose $\delta(p)=x \neq y$. Since $\mathrm{CT}_{3}^{\prime} \Longrightarrow \mathrm{T}_{3}$ and $\mathrm{T}_{3} \Longrightarrow \mathrm{T}_{2}$ for second countable spaces, the space is $T_{2}$, hence there is some $w$ such that $x \in \nu(w)$ and $y \notin \nu(w)$. Then on input $(p, w)$ the machine $M$ writes some $\iota\left(u_{1}\right) q$ such that $x \in \nu\left(u_{1}\right) \subseteq \psi^{-}(q) \subseteq \nu(w)$. Since $y \notin \nu(w)$, hence $y \in \theta(q)$, there are a prefix $v_{2}$ of $q$ and a word $v$ such that $v \ll v_{2}$ and $y \in \nu(v)$. For producing $\iota\left(u_{1}\right) v_{2}$ some prefix of $p$ is sufficient. Since $x \in \nu\left(u_{1}\right) \subseteq \nu(w)$ there is a prefix $v_{1}$ of $p$ such that $w \ll v_{1}, u_{1} \ll v_{1}$ and on input $\left(v_{1} 1^{\omega}, w\right)$ in length $\left(v_{1}\right)$ steps the machine $M$ writes at least $\iota\left(u_{1}\right) v_{2}$. Since $x \in \theta \circ g\left(v_{1}\right)$, there is some $u \ll g\left(v_{1}\right)$ such that $x \in \nu(u)$. By definition of $H,(u, v) \in H$, hence (3.2) is true.

Suppose $(u, v) \in H$. Then there are words $w, u_{1}, v_{1}, v_{2}$ with the properties listed in the definition of $H$. If $\nu(u)=\emptyset,(3.3)$ is true.

Suppose $x \in \nu(u) \neq \emptyset$. Since $u \ll g\left(v_{1}\right)$ and $w \ll v_{1}, x \in \nu(u) \subseteq \theta \circ g\left(v_{1}\right)=\bigcap \nu^{\text {fs }}\left(v_{1}\right) \subseteq$ $\nu(w)$. There is some $p^{\prime} \in \Sigma^{\omega}$ such that $x=\delta\left(v_{1} p^{\prime}\right) \in \bigcap \nu^{\mathrm{fs}}\left(v_{1}\right)$. Since $M$ realizes $t_{3}^{\prime}$, on input $\left(v_{1} p^{\prime}, w\right)$ the machine $M$ writes $\iota\left(u_{1}\right) q$ such that $x \in \nu\left(u_{1}\right) \subseteq \psi^{-}(q) \subseteq \nu(w)$. In $\operatorname{length}\left(v_{1}\right)$ steps the machine can read only symbols from $v_{1}$ and, therefore, has the same behavior on input $\left(v_{1} 1^{\omega}, w\right)$. By assumption on $u, v, w, u_{1}, v_{1}$ and $v_{2}, v_{2}$ is a prefix of $q$ and $u_{1} \ll v_{1}$, hence $\nu(u) \subseteq \nu\left(u_{1}\right)$. Since $v \ll v_{2}, v \ll q$, hence $\nu(v) \subseteq \theta(q)$. Since $\nu(u) \subseteq \nu\left(u_{1}\right) \subseteq \psi^{-}(q)$, $\nu(u) \cap \nu(v) \subseteq \nu\left(u_{1}\right) \cap \theta(q)=\emptyset$. Therefore, (3.3) is true.

$\mathrm{SCT}_{2} \Longrightarrow \mathrm{CT}_{2}$ : See [12]

$\mathbf{D} \Longrightarrow \mathbf{W C T}_{3}$ : See Example 3.3(4).

The statements (1) - (6) of the theorem follow from these results. 
By [12, Theorem 7], the following statements are equivalent: $\mathbf{X}$ is $S C T_{2} ; x \neq y$ is $(\delta, \delta)$-r.e.; $x \mapsto \overline{\{x\}}$ is $\left(\delta, \psi^{-}\right)$-computable. We apply this result in the next proof.

Theorem 4.2. $\mathrm{CT}_{4}+\mathrm{SCT}_{2} \Rightarrow \mathrm{CT}_{3}$.

Proof: Since the space is $S C T_{2}$ it is $T_{1}[12$, Theorem 5], hence $\{x\}=\overline{\{x\}}$ for every point $x$. Therefore by the above characterization, from $x$ and $A$ such that $x \notin A$ we can compute $\{x\}$ and $A$ and by $C T_{4}$ we can compute disjoint open sets $U, V$ such that $\{x\} \subseteq U$, hence $x \in U$, and $A \subseteq V$. Therefore, the space is $C T_{3}$.

By [12, Theorem 7], $\mathrm{T}_{2} \Rightarrow \mathrm{SCT}_{2}$ if $U \cap V=\emptyset$ is $(\nu, \nu)$-r.e.. A similar result holds for $T_{3}$-spaces.

Theorem 4.3. $\mathrm{CT}_{3} \Longleftrightarrow \mathrm{WCT}_{3}$ if $U \cap V=\emptyset$ is $(\nu, \nu)$-r.e.

Proof: Suppose $\mathrm{WCT}_{3}$. Then from $x \in X$ and $W \in \beta$ such that $x \in W$ we can compute some $U \in \beta$ such that $x \in U \in \bar{U} \in W$. For showing $\mathrm{CT}_{3}^{\prime}$ it suffices to find a $\psi^{-}$-name of $\bar{U}$. By assumption, from $U \in \beta$ we can find a list (encoded by $q \in \Sigma^{\omega}$ ) of all $V \in \beta$ such that $U \cap V=\emptyset$. Since for open $V, U \cap V=\emptyset \Longleftrightarrow \bar{U} \cap V=\emptyset, q$ is a $\psi^{-}$-name of the closed $\bar{U}$. Therefore, the space is $C T_{3}^{\prime}$, hence $C T_{3}$.

For a computable topological space $\mathbf{X}=(X, \tau, \beta, \nu)$ possibly $U=\emptyset$ for some $U \in \beta$.

Theorem 4.4. If the set $\left\{w \in \Sigma^{*} \mid \nu(w) \neq \emptyset\right\}$ is r.e. then

$$
\mathrm{CT}_{3} \Longleftrightarrow \mathrm{CTy} \Longleftrightarrow \mathrm{SCT}_{3} \text {. }
$$

In particular, if all base elements are not empty then $\mathrm{CT}_{3} \Longleftrightarrow \mathrm{SCTy} \Longleftrightarrow \mathrm{SCT}_{3}$. Of course, the space $\mathbf{X}$ in Example 5.4 has empty base elements. The non-empty ones are not even r.e.

Proof: Suppose that $\{w \mid \nu(w) \neq \emptyset\}$ is r.e. Since finite intersection is $\left(\nu^{\mathrm{fs}}, \theta\right)$-computable [13. Theorem 11], there is a computable function $g$ such that $\bigcap \nu^{\mathrm{fs}}(w)=\theta \circ g(w)$. Therefore, the set $\left\{w \in \Sigma^{*} \mid \bigcap \nu^{\mathrm{fs}}(w) \neq \emptyset\right\}$ is r.e. Suppose the space is $C T_{3}$. By Theorem 4.1 it is $C T_{3}^{\prime}$. There is a machine $M$ such that $f_{M}$ realizes the multi-function $t_{3}^{\prime}$ from $\mathrm{CT}_{3}^{\prime}$ in Definition 3.2 .

Let $x_{0}=\delta\left(p_{0}\right) \in \nu(w)$. Then for some $u \in \operatorname{dom}(\nu)$ and $q_{p_{0}} \in \operatorname{dom}\left(\psi^{-}\right), f_{M}\left(p_{0}, w\right)=$ $\left\langle u, q_{p_{0}}\right\rangle=\iota(u) q_{p_{0}}$ such that

$$
x_{0} \in \nu(u) \subseteq \psi^{-}\left(q_{p_{0}}\right) \subseteq \nu(w) .
$$

For computing $\iota(u)$ some prefix $u_{0} \in \operatorname{dom}\left(\nu^{\mathrm{fs}}\right) \cap \Sigma^{*} 11$ of $p_{0}$ suffices. Since $\delta\left(p_{0}\right) \in \nu(w)$ we may assume $w \ll u_{0}$. For all $p \in I_{0}:=\left\{p \in \operatorname{dom}(\delta) \mid u_{0}\right.$ is a prefix of $\left.p\right\}, f_{M}(p, w)=\iota(u) q_{p}$ for some $q_{p}$ such that $\delta(p) \in \nu(u) \subseteq \psi^{-}\left(q_{p}\right) \subseteq \nu(w)$. Then

$$
x_{0} \in \nu(u) \in \bigcap_{p \in I_{0}} \psi^{-}\left(q_{p}\right) \subseteq \nu(w) .
$$

A word $u_{0} \in \operatorname{dom}\left(\nu^{\mathrm{fs}}\right)$ is a prefix of some $p \in \operatorname{dom}(\delta)$ iff $\bigcap \nu^{\mathrm{fs}}\left(u_{0}\right) \neq \emptyset$. We will define $R$ such that $(u, w) \in R$ iff for some $u_{0} \in \Sigma^{*} 11$ such that $\bigcap \nu^{\mathrm{fs}}\left(u_{0}\right) \neq \emptyset$ the machine $M$ on input $\left(u_{0} 1^{\omega}, w\right)$ writes $\iota(u)$ in at most $\left|u_{0}\right|$ steps. From this word $u_{0}$ we will compute a sequence $q \in \Sigma^{\omega}$ such that $\psi^{-}(q)=\bigcap_{p \in I_{0}} \psi^{-}\left(q_{p}\right)$.

There is a machine $N$ that works on input $(u, w)$ as follows:

(S1) $N$ searches for some $u_{0} \in \operatorname{dom}\left(\nu^{\mathrm{fs}}\right) \cap \Sigma^{*} 11$ such that $w \ll u_{0}, \bigcap \nu^{\mathrm{fs}}\left(u_{0}\right) \neq \emptyset$ and the 
machine $M$ on input $\left(u_{0} 1^{\omega}, w\right)$ writes $\iota(u)$ in at most $\left|u_{0}\right|$ steps.

(S2) Then $N$ writes every $\iota(v)$ such that there are words $u^{\prime}$ and $v^{\prime}$ such that

(S2a) $u^{\prime} \in \operatorname{dom}\left(\nu^{\mathrm{fs}}\right) \cap \Sigma^{*} 11, \bigcap \nu^{\mathrm{fs}}\left(u^{\prime}\right) \neq \emptyset$ and $u_{0} \sqsubseteq u^{\prime}$ and

(S2b) $M$ on input $\left(u^{\prime} 1^{\omega}, w\right)$ in $\left|u^{\prime}\right|$ steps writes $\iota(u) v^{\prime}$ such that $v \ll v^{\prime}$.

Furthermore, $N$ writes 11 repeatedly in order to produce an infinite sequence if only finitely many words $v$ can be found. If no $u_{0}$ can be found the machine does not halt and writes nothing. Let $r:=f_{N}$ and $R:=\operatorname{dom}\left(f_{N}\right)$. Then $R \subseteq \operatorname{dom}(\nu) \times \operatorname{dom}(\nu)$ and $R$ is r.e. We must prove (3.4) and (3.5).

We show (3.4): Suppose $x=\delta(p) \in \nu(w)$. Then for some $u$ and $q, f_{M}(p, w)=\iota(u) q$ such that $x \in \nu(u) \subseteq \psi^{-}(q) \subseteq \nu(w)$. There is a prefix $u_{0} \in \Sigma^{*} 11$ of $p$ such that $w \ll u_{0}$ and $M$ on input $\left(u_{0} 1^{\omega}, w\right)$ writes $\iota(u)$ in at most $\left|u_{0}\right|$ steps, hence $(u, w) \in \operatorname{dom}\left(f_{N}\right)=R$. Therefore, $x \in \nu(u)$ for some $u$ with $(u, w) \in R$. We conclude $\nu(w) \subseteq \bigcup\{\nu(u) \mid(u, w) \in R\}$.

On the other hand, let $(u, w) \in R$. Then there is some $u_{0} \in \operatorname{dom}\left(\nu^{\mathrm{fs}}\right) \cap \Sigma^{*} 11$ such that $\bigcap \nu^{\mathrm{fs}}\left(u_{0}\right) \neq \emptyset$ and the machine $M$ on input $\left(u_{0} 1^{\omega}, w\right)$ writes $\iota(u)$ in at most $\left|u_{0}\right|$ steps. There is some $p^{\prime}$ such that $u_{0} p^{\prime} \in \operatorname{dom}(\delta)$. Then $f_{M}\left(u_{0} p^{\prime}, w\right)=\iota(u) q^{\prime}$ for some $q^{\prime}$ hence $\delta\left(u_{0} p^{\prime}\right) \in \nu(u) \subseteq \nu(w)$. Therefore, $\bigcup\{\nu(u) \mid(u, w) \in R\} \subseteq \nu(w)$.

Combining the two results we obtain (3.4).

We show (3.5): Suppose $(u, w) \in R=\operatorname{dom}\left(f_{N}\right)$ is the input of the machine $N$ and let $q:=f_{N}(u, w)$. First, $N$ finds some $u_{0}$ with the properties listed in (S1).

Suppose, later $N$ writes $\iota(v)$ as described in (S2). Then there are words $u^{\prime}, v^{\prime}$ and a sequence $p^{\prime} \in \Sigma^{\omega}$ such that $u^{\prime} p^{\prime} \in \operatorname{dom}(\delta)$ and $M$ on input $\left(u^{\prime} p^{\prime}, w\right)$ in at most $\left|u^{\prime}\right|$ steps writes $v^{\prime}$ such that $v \ll v^{\prime} \sqsubseteq q$ and $\delta\left(u^{\prime} p^{\prime}\right) \in \nu(u) \subseteq \nu(w)$, hence $\nu(u) \cap \nu(v)=\emptyset$. Therefore, $\nu(u) \cap \nu(v)=\emptyset$ for all $v$ such that $v \ll q$. We obtain $\nu(u) \subseteq \psi^{-}(q)=\psi^{-} \circ f_{N}(u, w)$.

There are some $p^{\prime}, q^{\prime}$ such that $u_{0} p^{\prime} \in \operatorname{dom}(\delta)$ and $M$ on input $\left(u_{0} p^{\prime}, w\right)$ writes $\iota(u) q^{\prime}$ such that $\delta\left(u_{0} p^{\prime}\right) \in \nu(u) \subseteq \psi^{-}\left(q^{\prime}\right) \subseteq \nu(w)$. Suppose, $v \ll q^{\prime}$. Then there are words $u^{\prime}, v^{\prime}$ such that the conditions (S2a) and (S2b) are satisfied, hence $v \ll f_{N}(u, w)$. Therefore, $\psi^{-} \circ f_{N}(u, w) \subseteq \psi^{-}\left(q^{\prime}\right) \subseteq \nu(w)$.

Combining the results we obtain $\nu(u) \subseteq \psi^{-} \circ f_{N}(u, w) \subseteq \nu(w)$. Therefore we have proved (3.5).

Notice that the proof works correctly since in (S1) we have guaranteed $\bigcap \nu^{\mathrm{fs}}\left(u_{0}\right) \neq \emptyset$ hence $u_{0} \sqsubseteq p$ for some $p \in \operatorname{dom}(\delta)$. The realization $f_{M}$ of $t_{3}^{\prime}$ may give unreasonable results on $(p, w)$ if $p \notin \operatorname{dom}(\delta)$.

\section{Counterexamples and Summary}

We show by counterexamples that some of the implications from Theorem 4.1 are proper. In [12] a $C T_{2}$-space is given that is not $S C T_{2}$, hence $\mathrm{SCT}_{2} \Longrightarrow \mathrm{CT}_{2}$ is proper.

Example 5.1. $\left(S C T_{2}\right.$ but not $\left.T_{3}\right)$ We extend [3, Example 1.5.7]. Let $\mathbf{R}=\left(\mathbb{R}, \tau_{\mathbb{R}}, \beta_{\mathbb{R}}, \nu_{\mathbb{R}}\right)$ be the computable real line from Example 3.3(1). Let $S:=\{1 / i \mid i \in \mathbb{Z}, i \neq 0\}, \sigma:=$ $\beta_{\mathbb{R}} \cup\{(-1 ; 1) \backslash S\}$ with canonical notation $\lambda$. Then $\mathbf{Z}=(\mathbb{R}, \sigma, \lambda)$ is a computable predicate space and $T(\mathbf{Z})=:(\mathbb{R}, \tau, \beta, \nu)$ is a computable topological space [13, Definition 8, Lemma 9]. Since $\beta_{\mathbb{R}}$ is a subset of the topology generated by $\sigma$ and $\nu_{\mathbb{R}} \leq \lambda$, " $x \neq y$ " is $\left(\delta_{\mathbf{Z}}, \delta_{\mathbf{Z}}\right)$-r.e., hence $(\delta, \delta)$-r.e. By [12, Theorem 7.2], $T(\mathbf{Z})$ is a $S C T_{2}$-space. The space is not $T_{3}$ since the point 0 cannot be separated from the closed set $S$ by disjoint open sets since $U \cap S \neq \emptyset$ for every neighborhood $U$ of 0 . 
Since the above $S C T_{2}$-space is not $T_{3}$ it is not $W C T_{3}, C T_{3}$ or $S C T_{3}$. The space from Example 5.3 below is $T_{4}$ and $S C T_{2}$, but not $W C T_{3}$. First we prove a lemma. Let us call a function $f: \subseteq \mathbb{Q} \rightarrow \mathbb{Q}$ a lower separation function for a real number $x>0$ if $f$ is computable (precisely, $\left(\nu_{\mathbb{Q}}, \nu_{\mathbb{Q}}\right)$-computable) and for all rational numbers $a$ with $0<a<x,(f(a)$ exists and) $a<f(a)<x$.

Lemma 5.2. There is a positive real number that has no lower separation function.

Proof: We define such a number $z$ by brute force diagonalization. Let $f_{1}, f_{2}, \ldots$ be a sequence of all computable partial functions $f: \subseteq \mathbb{Q} \rightarrow \mathbb{Q}$. Let $\left(a_{0} ; b_{0}\right):=(0 ; 1)$ and for $i=$ $1,2, \ldots$ define intervals $\left(a_{i} ; b_{i}\right)$ as follows. Find some rational number $a$ such that $a_{i-1}<a<$ $f_{i}(a)<b_{i-1}$ and define $\left(a_{i} ; b_{i}\right):=\left(a, f_{i}(a)\right)$, if no such $a$ exists define $\left(a_{i} ; b_{i}\right):=\left(a_{i-1} ; b_{i-1}\right)$. There is some positive real number $z \in \bigcap_{i}\left(a_{i} ; b_{i}\right)$. Suppose, $f_{k}$ is a lower separating function for $z$. Since $z \in\left(a_{k-1} ; b_{k-1}\right)$ there is some $a$ such that $a_{k-1}<a<f_{k}(a)<z<b_{k-1}$ and $a_{k}, b_{k}$ are chosen such that $a_{k-1}<a_{k}<f_{k}\left(a_{k}\right)<b_{k-1}$ and $b_{k}=f_{k}\left(a_{k}\right)$. If $a_{k}<z$ then $b_{k}=f_{k}\left(a_{k}\right)<z$, hence $z \notin\left(a_{k} ; b_{k}\right)$, if $z \leq a_{k}$ then $z \notin\left(a_{k} ; b_{k}\right)$ as well. But by assumption $z \in \bigcap_{i}\left(a_{i} ; b_{i}\right)$. Therefore, $f_{k}$ cannot be a lower separating function for $z$.

Example 5.3. ( $T_{4}$ and $S C T_{2}$ but not $\left.W C T_{3}\right)$ Let $\mathbf{R}=(\mathbb{R}, \tau, \beta, \nu)$ be the computable real line from Example 3.3(1). For $c \in \mathbb{R}$ define $\mathbf{R}_{c}=\left(\mathbb{R}, \tau, \beta_{c}, \nu_{c}\right)$ by $\nu_{c}(0 w):=\nu(w)$, and $\nu_{c}(1 w):=\nu(w) \cap(-\infty ; c)$. Then $\mathbf{R}_{c}$ is a computable topological space. Let $\delta_{c}$ be the (canonical or inner) representation of $\mathbb{R}$ for $\mathbf{R}_{c}$ [13, Definition 5.1]. Since $\mathbf{R}_{c}$ has the same topology as $\mathbf{R}$ it is $T_{i}$ for $i=0, \ldots, 4$. The computable real line $\mathbf{R}$ is $S C T_{2}$. Let $H$ satisfy (3.2) and (3.3) for $\mathbf{R}$. Then $H_{c}:=\{(0 v, 0 w) \mid(v, w) \in H\}$ satisfies (3.2) and (3.3) for $\mathbf{R}_{c}$. Therefore, $\mathbf{R}_{c}$ is $S C T_{2}$.

Let $c>0$ be a real number that has no lower separation function (Lemma 5.2). Suppose $\mathbf{R}_{c}$ is $W C T_{3}$. Let $t_{3}^{w}$ be the computable function from Definition 3.2 for $\mathbf{R}_{c}$. Then:

- the function $a \mapsto a$ for rational $0<a<c$ is $\left(\nu_{\mathbb{Q}}, \delta_{c}\right)$-computable,

$-\nu_{c}\left(w_{0}\right)=(0 ; c)$ for some $w_{0} \in \Sigma^{*}$,

- for every $x \in(0 ; c), t_{3}^{w} \operatorname{maps}(x,(0 ; c))$ to some $U \in \beta_{c}$ such that $x \in U \subseteq \bar{U} \subseteq(0 ; c)$.

- $U \mapsto \sup U$ for $U \in \beta_{c}$ such that $\bar{U} \subseteq(0 ; c)$ is $\left(\nu_{c}, \nu_{\mathbb{Q}}\right)$-computable.

There is a $\left(\nu_{\mathbb{Q}}, \nu_{\mathbb{Q}}\right)$-computable multi-function $h$ mapping each rational number $0<$ $a<c$ to some rational number $b$ such that $a<b<c$ : From a $\nu_{\mathbb{Q}^{-n a m e}}$ of $a$ compute a $\delta_{c}$-name of $a$. By $t_{3}^{w}$, from $a$ and $(0 ; c)$ compute some $U \in \beta_{c}$ such that $a \in U \subseteq \bar{U} \subseteq(0 ; c)$. From $U$ compute $b:=\sup U \in \mathbb{Q}$. Then $a<b<c$. Since there is an injective notation equivalent to $\nu_{\mathbb{Q}}$, the function $h$ is single-valued. Therefore, $h$ is a lower separation function for $c$. Contradiction.

The above space is $T_{4}, T_{3}$ and $S C T_{2}$ but not $W C T_{3}, C T_{3}$ or $S C T_{3}$. Finally we separate CTy from $\mathrm{SCT}_{3}$. (The example in [11] in [1] for separating $\mathrm{CT}_{3}$ from $\mathrm{SCT}_{3}$ is not correct.)

Example 5.4. (D and CTy but not $\mathrm{SCT}_{3}$ ) Let $X:=\mathbb{N}$ and let $A \subseteq \mathbb{N}$ be the set defined below. Let $\tau$ be the discrete topology on $\mathbb{N}$ and define a notation $\nu$ of a base $\beta$ of $\tau$ by $\nu\left(12^{j}\right):=\{j\}, \nu(2):=A$ and $\nu\left(02^{j}\right):=\{j\} \cap A$. Then $\mathbf{X}:=(\mathbb{N}, \tau, \beta, \nu)$ is a computable topological space. Let $\delta$ be the canonical representation of the points of $\mathbf{X}[13$.

We show that $\mathbf{X}$ is $C T y^{\prime}$.

Let $p_{0}, p_{1} \in \Sigma^{\omega}$ be computable sequences such that $\rho\left(p_{0}\right)=0 \in \mathbb{R}$ and $\rho\left(p_{1}\right)=1 \in \mathbb{R}$. There is a machine $M$ that in input $(p, w, q) \in \Sigma^{\omega} \times \Sigma^{*} \times \Sigma^{\omega}$ searches for $i, j \in \mathbb{N}$ such that $12^{i} \ll p$ and $12^{j} \ll q$ and then writes $p_{0}$ if $i=j$ and $p_{1}$ else. Then for all $p, q \in \operatorname{dom}(\delta)$ 
and all $w \in \Sigma^{*}, \rho \circ f_{M}(p, w, q)=(0$ if $\delta(p)=\delta(q)$ and 1 else $)$. Therefore, $f_{M}$ realizes the function $f: X \times \beta \times X \rightarrow \mathbb{R}$ such that $f(x, W, y)=(0$ if $x=y$ and 1 else). By type conversion [9, Theorem 3.3.15] the function $(x, W) \mapsto f$ such that $f(y)=(0$ if $x=y$ and 1 else) is $(\delta, \nu,[\delta \rightarrow \rho])$-computable. Furthermore, from $p \in \operatorname{dom}(\delta)$ we can compute the (unique) $i$ such that $12^{i} \ll p$. Then $\delta(p)=i$ and $\{i\}=\nu\left(12^{i}\right)=: U$. Therefore, from $x$ and $W$ such that $x \in W$ we can find $U$ and $f$ such that the conditions for $\mathrm{CTy}^{\prime}$ hold true.

We define $A \subseteq \mathbb{N}$. Let $K \subseteq \mathbb{N}$ be a set with non r.e. complement. Let $A \cap(2 \mathbb{N}+1):=$ $2 K+1$. Define $A \cap 2 \mathbb{N}$ as follows. Let $\gamma_{i}$ be the $i$ th computable function $f: \subseteq \Sigma^{*} \times \Sigma^{*} \rightarrow \Sigma^{*}$ $(i=0,1,2, \ldots)$ and let $\lambda_{i}$ be the $i$ th computable function $f: \subseteq \Sigma^{*} \times \Sigma^{*} \rightarrow \Sigma^{\omega}$. For $n=\langle i, k\rangle$ $(\langle\rangle$ is the Cantor pairing function) define the position of $2 n$ as follows by diagonalization.

$$
\begin{aligned}
\text { if } & \left(12^{2 n}, 2\right) \notin \operatorname{dom}\left(\gamma_{i}\right) \text { and }\left(02^{2 n}, 2\right) \notin \operatorname{dom}\left(\gamma_{i}\right) \quad \text { then } \quad \begin{array}{l}
2 n \in A, \\
\text { then }
\end{array} 2 n \notin A, \\
\text { if }\left(12^{2 n}, 2\right) \in \operatorname{dom}\left(\gamma_{i}\right) & \\
\text { if }\left(12^{2 n}, 2\right) \notin \operatorname{dom}\left(\gamma_{i}\right) \text { and }\left(02^{2 n}, 2\right) \in \operatorname{dom}\left(\gamma_{i}\right) &
\end{aligned}
$$

Suppose, $\mathbf{X}$ is $S C T_{3}$. Let $R$ be the r.e. set and let $r$ be the computable function such that (3.4) and (3.5) hold true. Then there are $i, k \in \mathbb{N}$ such that $R=\operatorname{dom}\left(\gamma_{i}\right)$ and $r=\lambda_{k}$.

Suppose, $(2,2) \in \operatorname{dom}\left(\gamma_{i}\right)$. Then by (3.5), $A=\nu(2) \subseteq \psi^{-} \circ \lambda_{k}(2,2) \subseteq \nu(2)$, hence $\mathbb{N} \backslash A=\bigcup\{\nu(v) \mid v \in V\}$ for an r.e. set $V \subseteq \operatorname{dom}(\nu)$. Since $2 \notin V$ and $\nu\left(02^{l}\right)=\emptyset$ for $l \notin A, m \notin K \Longleftrightarrow 2 m+1 \notin A \Longleftrightarrow 12^{2 m+1} \in V$, hence the complement of $K$ is r.e. (contradiction). Therefore, $(2,2) \notin \operatorname{dom}\left(\gamma_{i}\right)$.

We show that $\gamma_{i}$ and $\lambda_{k}$ cannot operate correctly for $n:=\langle i, k\rangle$.

Case $\left(12^{2 n}, 2\right) \notin \operatorname{dom}\left(\gamma_{i}\right)$ and $\left(02^{2 n}, 2\right) \notin \operatorname{dom}\left(\gamma_{i}\right)$ : Since $(2,2) \notin \operatorname{dom}\left(\gamma_{i}\right), 2 n \notin \nu(2)$ by (3.4). But $2 n \in A=\nu(2)$ by the definition of $A$. Contradiction.

Case $\left(12^{2 n}, 2\right) \in \operatorname{dom}\left(\gamma_{i}\right)$ : Then $2 n \in A=\nu(2)$ by (3.4). But $2 n \notin A=\nu(2)$ by the definition of $A$. Contradiction.

Case $\left(12^{2 n}, 2\right) \notin \operatorname{dom}\left(\gamma_{i}\right)$ and $\left(02^{2 n}, 2\right) \in \operatorname{dom}\left(\gamma_{i}\right):$ By (3.4),

$$
\{2 n\} \cap A=\nu\left(02^{2 n}\right) \subseteq \psi^{-} \circ \lambda_{k}\left(02^{2 n}, 2\right) \subseteq \nu(2)=A .
$$

Suppose $2 n \in A$. Then $2 n \in \psi^{-} \circ \lambda_{k}\left(02^{2 n}, 2\right) \subseteq A$, hence $2 n \notin \theta \circ \lambda_{k}\left(02^{2 n}, 2\right)$. Therefore, $02^{2 n} \nless \lambda_{k}\left(02^{2 n}, 2\right)$ and $12^{2 n} \nless \lambda_{k}\left(02^{2 n}, 2\right)$. Then $2 n \notin A$ by the definition of $A$. Contradiction.

Suppose $2 n \notin A$. By (5.1), $2 n \in \theta \circ \lambda_{k}\left(02^{2 n}, 2\right)$, hence $02^{2 n} \ll \lambda_{k}\left(02^{2 n}, 2\right)$ or $12^{2 n} \ll$ $\lambda_{k}\left(02^{2 n}, 2\right)$. Then $2 n \in A$ by the definition of $A$. Contradiction.

Therefore, the space $\mathbf{X}$ is not $\mathrm{SCT}_{3}$.

We summarize the counterexamples.

Theorem 5.5. The following implications are false:

$$
\begin{array}{rlll}
C T_{2}+D & \Longrightarrow & S C T_{2} & ([12, \text { Example 5]), } \\
C T_{0}+C T_{4} & \Longrightarrow & T_{1} & \text { (Example [3.3(3)) } \\
S C T_{2} & \Longrightarrow & T_{3} & \text { (Example 5.1), } \\
S C T_{2}+T_{4} & \Longrightarrow & W C T_{3} & \text { (Example 5.3), } \\
C T y+D & \Longrightarrow & S C T_{3} & \text { (Example 5.4). }
\end{array}
$$


Further false implications can be obtained by transitivity of " $\Longrightarrow "$, for example, $\mathrm{CT}_{4} \Longrightarrow \mathrm{SCT}_{3}$ is false by (5.3) since $\mathrm{SCT}_{3} \Longrightarrow \mathrm{T}_{1}$. Figure 1 shows the positive and negative results that we have proved. " $A \longrightarrow B$ " means $A \longrightarrow B$, , $A \stackrel{C}{\longrightarrow} B$ " means $A \wedge C \Longrightarrow B$, " $A \nrightarrow B$ " means that we have constructed a computable topological space for which $A \wedge \neg B$, and " $A \stackrel{C}{\longrightarrow} B$ " means that we have constructed a computable topological space for which $(A \wedge C) \wedge \neg B$. EI abbreviates " $U \cap V=\emptyset$ is $(\nu, \nu)$-r.e." and NE abbreviates " $U \neq \emptyset$ is $\nu$-r.e.".

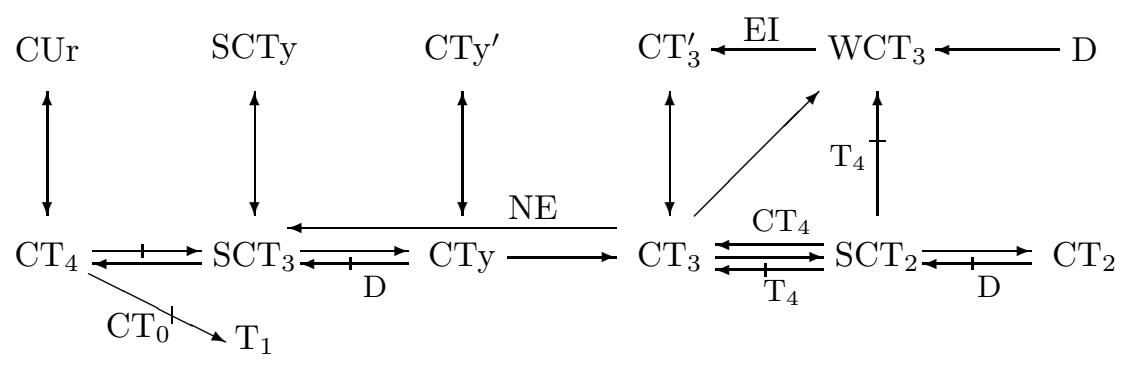

Figure 1: Logical relations between the computable separation axioms.

A number of implications have not yet been proved or disproved, for example,

$$
\begin{aligned}
W C T_{3} & \Longrightarrow C T_{3}\left(S C T_{2}, C T_{2}, C T_{0}, W C T_{0}\right), \\
C T_{3} & \Longrightarrow C T y\left(C T_{4}\right), \\
C T_{4}+T_{3} & \Longrightarrow\left(S C T_{3}, C T_{3}, S C T_{2}, C T_{2}, C T_{0}, W C T_{0}\right), \\
C T_{4}+S C T_{2} & \Longrightarrow S C T_{3}(C T y) .
\end{aligned}
$$

(The axioms $\mathrm{CT}_{0}$ and $\mathrm{WCT}_{0}$ are defined in [12].) A difficulty arises from the fact that for $T_{1}$-spaces (where the singleton sets are closed) the function $x \mapsto\{x\}$ is $\left(\delta, \psi^{+}\right)$-computable but in general not $\left(\delta, \psi^{-}\right)$-computable. In our computable separation axioms, however, we use only the outer representation $\psi^{-}$for the closed sets.

\section{Computable Metrization}

For a metric space $(X, d)$ the open balls with rational radius and center from a dense set are a basis of a topology, the topology generated by it [3]. A topological space $(X, \tau)$ is metrizable, iff it is generated by a metric space. A second countable space is metrizable iff it is $T_{3}$ (remember $\mathrm{T}_{3} \Longrightarrow \mathrm{T}_{2}$ ) [3] Theorem 4.2.9]. Every second countable metrizable space has an at most countable dense subset $A$. Therefore, it can be enriched by a notation $\alpha$ of this dense set. The following definitions are essentially from [8, 9].

\section{Definition 6.1.}

(1) An effective metric space is a tuple $\mathbf{M}=(X, d, A, \alpha)$ such that $(M, d)$ is a metric space and $\alpha$ is a notation of a set $A \subseteq X$ which is dense in $X$.

(2) The Cauchy representation $\delta_{C}$ of an effective metric space $\mathbf{M}$ is defined by $\delta_{C}(p)=x$ iff there are words $u_{0}, u_{1}, \ldots \in \operatorname{dom}(\alpha)$ such that $p=\iota\left(u_{0}\right) \iota\left(u_{1}\right) \ldots$ and $d\left(x, \alpha\left(u_{i}\right)\right) \leq 2^{-i}$ for all $i \in \mathbb{N}$.

(3) The effective topological space [13, Definition 4] associated with the effective metric space is the tuple $\mathbf{X}=(X, \tau, \beta, \nu)$ such that $\nu, \nu\langle u, s\rangle:=B\left(\alpha(u), \nu_{\mathbb{Q}}(s)\right)$, is the canonical notation of the set $\beta$ of all open balls with center from $A$ and rational radius and $\tau$ is the smallest topology containing $\beta$. 
(4) An upper semi-computable (lower semi-computable) metric space is an effective metric space such that $\operatorname{dom}(\alpha)$ is recursive and $d(a, b)<s(s<d(a, b))$ is $\left(\alpha, \alpha, \nu_{\mathbb{Q}}\right)$-r.e.

(5) A computable metric space is an effective metric space such that $\operatorname{dom}(\alpha)$ is recursive and $r<d(a, b)<s$ is $\left(\nu_{\mathbb{Q}}, \alpha, \alpha, \nu_{\mathbb{Q}}\right)$-r.e.

Notice that $d(a, b)<s$ is $\left(\alpha, \alpha, \nu_{\mathbb{Q}}\right)$-r.e. iff the distance on $A$ is $\left(\alpha, \alpha, \rho_{>}\right)$-computable iff $d$ is $\left(\delta_{C}, \delta_{C}, \rho_{>}\right)$-computable [13, Example 1] $\left[9\right.$, and $r<d(a, b)<s$ is $\left(\nu_{\mathbb{Q}}, \alpha, \alpha, \nu_{\mathbb{Q}}\right)$ r.e. iff the distance on $A$ is $(\alpha, \alpha, \rho)$-computable iff the distance is $\left(\delta_{C}, \delta_{C}, \rho\right)$-computable (Example 3.3(1) ) [9]. Since for every notation $\alpha: \subseteq \Sigma^{*} \rightarrow A$ with r.e. domain there is a notation $\alpha^{\prime}: \subseteq \Sigma^{*} \rightarrow A$ with recursive domain such that $\alpha \equiv \alpha^{\prime}$, allowing r.e. domains in Definition 6.1(4) and (5) is no proper generalization.

Theorem 6.2. For every effective metric space $\mathbf{M}=(X, d, A, \alpha)$ with Cauchy representation $\delta_{C}$ and its associated effective topological space $\mathbf{X}=(X, \tau, \beta, \nu)$ with canonical representation $\delta$,

(1) if $\mathbf{M}$ is upper semi-computable, then $\mathbf{X}$ is a computable topological space.

(2) $\delta \leq \delta_{C} ; \delta_{C} \leq_{t} \delta ; \delta_{C} \leq \delta$ if $\mathbf{M}$ is upper semi-computable,

(3) if $\mathbf{M}$ is a computable metric space, then all the separation axioms from Definition 3.2 hold true for $\mathbf{X}$.

The first two items of this theorem differ slightly from [9, Theorem 8.1.4] since computable topological space is defined differently.

Proof: (11) We must show that intersection is $(\nu, \nu, \theta)$-computable. Observe that $B\left(a_{1}, r_{1}\right) \cap B\left(a_{2}, r_{2}\right)=\bigcup\left\{B(a, r) \mid d\left(a_{1}, a\right)<r_{1}-r \wedge d\left(a_{2}, a\right)<r_{2}-r\right\}$. Since $d(a, b)<s$ is r.e., there is an r.e. set $S$ such that $\nu(u) \cap \nu(v)=\bigcup\{\nu(w) \mid(u, v, w) \in S\}$.

(2) If $\delta(p)=x$, then $p$ is a list of all $\langle u, v\rangle$ such that $d(x, \alpha(u))<\nu_{\mathbb{Q}}(v)$. Therefore, there is a machine $M$ which from $p \in \operatorname{dom}(\delta)$ computes a sequence $\iota\left(u_{0}\right) \iota\left(u_{1}\right) \ldots$ such that for all $i, d\left(x, \alpha\left(u_{i}\right)\right) \leq 2^{-i}$.

Now let $\mathbf{M}$ be upper semi-computable. If $\delta_{C}(p)=x$ then $p=\iota\left(u_{0}\right) \iota\left(u_{1}\right) \ldots$ such that for all $i, d\left(x, \alpha\left(u_{i}\right)\right) \leq 2^{-i}$. Observe that $x \in B(a, r) \Longleftrightarrow(\exists i) d\left(a, \alpha\left(u_{i}\right)\right)<r-2^{-i}$. Since $d(a, b)<s$ is r.e., from $p$ we can compute a list of all $w$ such that $x \in \nu(w)$.

If $\mathbf{M}$ is not upper semi-computable then there are "oracles" $q, q^{\prime} \in \Sigma^{\omega}$ such that $\operatorname{dom}(\alpha)$ is recursive in $q$ and $d(a, b)<s$ is r.e. in $q^{\prime}$. Using the oracles $q, q^{\prime}$, there is a machine translating $\delta_{C}$ to $\delta$. The function $f_{M}$ computed by this machine is continuous [9].

(3) By Theorem 4.1 it suffices to prove $\mathrm{SCT}_{3}$. Let $R:=\left\{\left(\langle u, v\rangle,\left\langle u^{\prime}, v^{\prime}\right\rangle\right) \mid\langle u, v\rangle,\left\langle u^{\prime}, v^{\prime}\right\rangle \in\right.$ $\left.\operatorname{dom}(\nu), \quad d\left(\alpha(u), \alpha\left(u^{\prime}\right)\right)+\nu_{\mathbb{Q}}(v)<\nu_{\mathbb{Q}}\left(v^{\prime}\right)\right\}$. Then (3.4) and $\overline{\nu(\langle u, v\rangle)} \subseteq \nu\left(\left\langle u^{\prime}, v^{\prime}\right\rangle\right)$

for $\left(\langle u, v\rangle,\left\langle u^{\prime}, v^{\prime}\right\rangle\right) \in R$. We compute a $\psi^{-}$-name of this closure. There is a machine that on input $\left(\langle u, v\rangle,\left\langle u^{\prime}, v^{\prime}\right\rangle\right) \in R$ lists all $\left\langle w, w^{\prime}\right\rangle \in \operatorname{dom}(\nu)$ such that $d(\alpha(u), \alpha(w))>$ $\nu_{\mathbb{Q}}(v)+\nu_{\mathbb{Q}}\left(w^{\prime}\right)$. Then (3.5) holds true for the function $r:=f_{M}$.

Since for an effective topological space $(X, \tau, \beta, \nu), \delta$ is an admissible representation, by Theorem 6.2(2) the Cauchy representation is admissible, that is, it is continuous and $\delta \leq_{t} \delta_{C}$ for every continuous representation of $X[9]$.

In general, we are interested in metric spaces $(X, d)$ with representation $\delta: \subseteq \Sigma^{\omega} \rightarrow X$ such that the distance is at least $\left(\delta, \delta, \rho_{>}\right)$-continuous. In this case the metric space is separable and the representation $\delta: \subseteq \Sigma^{\omega} \rightarrow X$ is continuous [9, Lemma 8.1.1]. By adding a notation of a dense set we obtain an effective metric space with Cauchy representation $\delta_{C}$. Then $\delta \leq_{t} \delta_{C}$, since the Cauchy representation is admissible. 
We call a metric on a computable topological space $\mathbf{X}=(x, \tau, \beta, \nu)$ with canonical representation $\delta$ of the points lower semi-computable, if it is $\left(\delta, \delta, \rho_{<}\right)$-computable and computable, if it is $(\delta, \delta, \rho)$-computable.

Theorem 6.3. Let $\mathbf{X}$ be a computable topological space.

(1) Suppose some lower semi-computable metric d generates the topology of $\mathbf{X}$. Then $\mathbf{X}$ is $S C T_{2}$.

(2) [7, 4] Suppose $\mathbf{X}$ is $S C T_{3}$. Then its topology is generated by some computable metric.

Theorem 6.3(2) has been proved in [7]. The shorter proof in [4] assumes $U \neq \emptyset$ for $U \in \beta$ but actually does not need this condition. We include a proof, since parts of it will be used in the proof of the next theorem.

\section{Proof:}

(11) By [13, Theorem 11] there is a computable function $g$ such that $\bigcap \nu^{\mathrm{fs}}(w)=\theta \circ g(w)$. There is a machine $M$ such that $f_{M}$ realizes the distance function w.r.t. $\left(\delta, \delta, \rho_{<}\right)$. Let $H$ be the set of all $(u, v)$ for which there are $v_{1}, v_{2} \in \operatorname{dom}\left(\nu^{\mathrm{fs}}\right)$ and $v_{3}, v_{4}$ such that

(a) the machine $M$ on input $\left(v_{1} 1^{\omega}, v_{2} 1^{\omega}\right)$ writes in at most $\max \left(\left|v_{1}\right|,\left|v_{2}\right|\right)$ steps the word $v_{3}, v_{4} \ll v_{3}$ and $\nu_{\mathbb{Q}}\left(v_{4}\right)>0$, and

(b) $u \ll g\left(v_{1}\right)$ and $w \ll g\left(v_{2}\right)$.

The set $H$ is r.e. We prove (3.2) and (3.3).

Suppose $\delta(p)=x \neq y=\delta(q)$. Since $d(x, y)>0$ there are $v_{1} \sqsubseteq p, v_{2} \sqsubseteq q, v_{3}$ and $v_{4}$ such that the machine $M$ on input $\left(v_{1} 1^{\omega}, v_{2} 1^{\omega}\right)$ writes in at most $\max \left(\left|v_{1}\right|,\left|v_{2}\right|\right)$ steps the word $v_{3}, v_{4} \ll v_{3}$ and $\nu_{\mathbb{Q}}\left(v_{4}\right)>0$. Since $x \in \bigcap \nu^{\mathrm{fs}}\left(v_{1}\right)$ and $y \in \bigcap \nu^{\mathrm{fs}}\left(v_{2}\right)$, there are $u \ll g\left(v_{1}\right)$ and $w \ll g\left(v_{2}\right)$ such that $x \in \nu(u)$ and $y \in \nu(w)$. By the definition of $H,(u, w) \in H$. This proves (3.2).

Suppose $(u, w) \in H$. If $\nu(u)=\emptyset$ or $\nu(w)=\emptyset$, then $\nu(u) \cap \nu(w)=\emptyset$. Suppose $\nu(u) \neq \emptyset$ and $\nu(w) \neq \emptyset$. Then there are words $v_{1}, v_{2}, v_{3}$ and $v_{4}$ such that, the machine $M$ on input $\left(v_{1} 1^{\omega}, v_{2} 1^{\omega}\right)$ writes in at $\operatorname{most} \max \left(\left|v_{1}\right|,\left|v_{2}\right|\right)$ steps the word $v_{3}, v_{4} \ll v_{3}, \nu_{\mathbb{Q}}\left(v_{4}\right)>0$, $u \ll g\left(v_{1}\right)$ and $w \in g\left(v_{2}\right)$. Since $\nu(u) \subseteq \bigcap \nu^{\mathrm{fs}}\left(v_{1}\right)$ and $\nu(w) \subseteq \bigcap \nu^{\mathrm{fs}}\left(v_{2}\right)$, for every $x \in \nu(u)$ and every $y \in \nu(w)$ there are sequences $p^{\prime}, q^{\prime}$ such that $x=\delta\left(v_{1} p^{\prime}\right)$ and $y=\delta\left(v_{2} q^{\prime}\right)$. Then the machine $M$ on input $\left(v_{1} p^{\prime}, v_{2} q^{\prime}\right)$ writes in at most $\max \left(\left|v_{1}\right|,\left|v_{2}\right|\right)$ steps the word $v_{3}$ such that $v_{4} \ll v_{3}$ and $\nu_{\mathbb{Q}}\left(v_{4}\right)>0$. Therefore, $d(x, y)>0$ for every $x \in \mu(u)$ and $y \in \nu(w)$. This proves (3.3).

(2) Since $R=\operatorname{dom}(r)$ is r.e., it has a computable numbering $\left(u_{i}, v_{i}\right)_{i \in \mathbb{N}}$. By Theorem 4.1, the Urysohn multi-function $t_{\text {Ur }}$ has a computable $\left(\psi^{-}, \psi^{-},[\delta \rightarrow \rho]\right)$-realization $h: \subseteq \Sigma^{\omega} \times$ $\Sigma^{\omega} \rightarrow \Sigma^{\omega}$.

The function $U \mapsto U^{c}$ for $U \in \beta$ has a computable $\left(\nu, \psi^{-}\right)$-realization $g: \subseteq \Sigma^{*} \rightarrow \Sigma^{\omega}$. For $i \in \mathbb{N}$ define $f_{i}: X \rightarrow \mathbb{R}, d_{i}: X \times X \rightarrow \mathbb{R}$ and $d: X \times X \rightarrow \mathbb{R}$ by

$$
\begin{aligned}
f_{i} & :=[\delta \rightarrow \rho] \circ h\left(r\left(u_{i}, v_{i}\right), g\left(v_{i}\right)\right) \\
d_{i}(x, y) & :=\left|f_{i}(x)-f_{i}(y)\right| \\
d(x, y) & :=\sum_{i} 2^{-i} d_{i}(x, y)
\end{aligned}
$$

Then for every $i, f_{i}$ is a continuous function such that range $\left(f_{i}\right) \subseteq[0 ; 1], f(x)=0$ for $x \in \nu\left(u_{i}\right)$ and $f(x)=1$ for $x \notin \nu\left(v_{i}\right)$, and $d_{i}$ is a continuous pseudometric on $(X, \tau)$ bounded by 1 such that $d_{i}(x, y)=1$ for $x \in \nu\left(u_{i}\right)$ and $y \notin \nu\left(v_{i}\right)$. 
Let $A$ be closed and non-empty and $x \notin A$. Then there is some $i$ such that $A \subseteq\left(\nu\left(v_{i}\right)\right)^{c}$ and $x \in \nu\left(u_{i}\right) \subseteq \nu\left(v_{i}\right)$. Then $d_{i}(x, A):=\inf \left\{a \in A \mid d_{i}(x, a)\right\}=1$. By [3, Lemma 4.4.6], $d$ is a metric which generates the topology $\tau$.

Since $i \mapsto f_{i}$ is $\left(\nu_{\mathbb{N}},[\delta \rightarrow \rho]\right)$-computable, the metric $d$ is $(\delta, \delta, \rho)$-computable.

The condition in the metrization theorem 6.3(2) is $S C T_{3}$. We do not know whether $S T y$ or $C T_{3}$ are sufficient to prove the metrization theorem.

For a computable metric space a dense set of computable points is needed. In general a space with computable metric does not have computable points but its metric completion may have computable points (example: the restriction of the computable real line (Example 3.3(10) to the non-computable real numbers). We will show that the metric space constructed in the proof of Theorem 6.3 can be completed to a computable metric space, if $\{u \in \operatorname{dom}(\nu) \mid \nu(u) \neq \emptyset\}$ is r.e..

For a pseudo-metric $d$ and sets $A, B$ we define the diameter and the distance of sets as usual: $\operatorname{dm}(A):=\sup \{d(x, y) \mid x, y \in A\}, d(A, B):=\inf \{d(x, y) \mid x \in A, y \in B\}$. The triangle inequality generalizes to

$$
d(A, C) \leq d(A, B)+d(B, C)+\operatorname{dm}(A)+\operatorname{dm}(B)+\operatorname{dm}(C) .
$$

We define a computable version of homeomorphic embedding [3, Section 2.1]. We will construct a computable metric space such that original computable topological space can be computably embedded into it.

Definition 6.4. For represented spaces $(X, \delta)$ and $\left(X^{\prime}, \delta^{\prime}\right)$, a computable embedding is an injective function $f: X \rightarrow X^{\prime}$ such that $f$ is $\left(\delta, \delta^{\prime}\right)$-computable and $f^{-1}$ is $\left(\delta^{\prime}, \delta\right)$-computable.

For computable topological spaces the standard representations are admissible, hence relatively computable functions are continuous. In this case a computable embedding is a homeomorphic embedding.

Theorem 6.5. 4] Let $\mathbf{X}=(X, \tau, \beta, \nu)$ be a computable topological space such that $\mathrm{CT}_{3}$ is true and the set $\{u \in \operatorname{dom}(\nu) \mid \nu(u) \neq \emptyset\}$ is r.e. Then there is a computable embedding of $\mathbf{X}$ into a computable metric space $\mathbf{M}=\left(M, d_{M}, A, \alpha\right)$ (where for $\mathbf{X}$ we consider the standard representation and for $\mathbf{M}$ the Cauchy representation).

In [4] the theorem has been proved for $S C T_{3}$ spaces with non-empty base sets. First we show that the assumptions in Theorem 6.5 are sufficient and then present a proof that uses ideas from [4] but is more transparent and much simpler.

Proof: By [13, Lemma 25] there is a computable topological space $\mathbf{X}^{\prime}=\left(X, \tau, \beta^{\prime}, \nu^{\prime}\right)$ equivalent to $\mathbf{X}$ such that $\nu^{\prime}(u) \neq \emptyset$ for all $u \in \operatorname{dom}\left(\nu^{\prime}\right)$. By [13, Theorem 22] equivalent means $\delta \equiv \delta^{\prime}$, hence the identity is a computable embedding of $\mathbf{X}$ into $\mathbf{X}^{\prime}$. By Lemma 3.4 we may assume w.l.o.g. that $\nu(u) \neq \emptyset$ for all $u \in \operatorname{dom}(\nu)$.

By Theorem 4.4 the space $\mathbf{X}$ is $S C T_{3}$. For $i \in \mathbb{N}$ let $f_{i}$ be the level function and $d_{i}$ the pseudo-metric and let $d$ be the metric defined in the proof of Theorem 6.3 ([6.1) , (6.2), $(\underline{6.3})$ ) with diameters $\mathrm{dm}_{i}$ and $\mathrm{dm}$, respectively. Remember that $i \mapsto f_{i}$ is $\left(\nu_{\mathbb{N}},[\delta \rightarrow \rho]\right)$ computable.

In the following we use nested sequences of non-empty open sets instead of Cauchysequences of points for completion. 


\section{Proposition 6.6.}

(1) The multi-function $g_{1}$ mapping every $(W, i, n)$ such that $W \in \beta$ and $i, n \in \mathbb{N}$ to some $(U, a)$ such that $U \in \beta, a \in \mathbb{Q}, U \subseteq W$ and $f_{i}[U] \subseteq\left(a-2^{-n} ; a+2^{-n}\right)$ is computable.

(2) The multi-function $g_{2}$ mapping every $(x, W, i, n)$ such that $x \in W \in \beta$ and $i, n \in \mathbb{N}$ to some $(U, a)$ such that $x \in U \in \beta, a \in \mathbb{Q}, U \subseteq W$ and $f_{i}[U] \subseteq\left(a-2^{-n} ; a+2^{-n}\right)$ is computable.

Proof: (Proposition 6.6)

(11) By the statement $\overrightarrow{\delta_{1}} \equiv \overrightarrow{\delta_{3}}$ in [13, Theorem 29] and (6.1) and since intersection on open sets is computable [13, Theorem 11], there is a computable function $g$ mapping $\left(i, n, a, u^{\prime}\right)\left(i, N \in \mathbb{N}, a \in \mathbb{Q}, u^{\prime} \in \operatorname{dom}(\nu)\right)$ to some $q \in \Sigma^{\omega}$ such that $f_{i}^{-1}\left[\left(a-2^{-n} ; a+\right.\right.$ $\left.\left.2^{-n}\right)\right] \cap \nu\left(u^{\prime}\right)=\theta(q)$. There is a machine $M$ that on input $(w, i, n)$ searches for some $a \in \mathbb{Q}$ and $u, u^{\prime} \in \operatorname{dom}(\nu)$ such that $\left(u^{\prime}, w\right) \in R, \nu(u) \neq \emptyset$ and $u \ll g\left(i, n, a, u^{\prime}\right)$ and then writes $(u, a)$.

There is some $y \in W=\nu(w)$. Then there is some $u^{\prime}$ such that $\left(u^{\prime}, w\right) \in R$ and $y \in \nu\left(u^{\prime}\right)$. There is some $a$ such that $f_{i}(y) \in\left(a-2^{-n} ; a+2^{-n}\right)$. Since $y \in f_{i}^{-1}\left[\left(a-2^{-n} ; a+2^{-n}\right)\right] \cap \nu\left(u^{\prime}\right)$ there is some $u$ such that $u \ll g\left(i, n, a, u^{\prime}\right)$. Therefore, the machine $M$ on input $(w, i, n)$ succeeds to write some $(u, a)$. In this case, $\nu(u) \subseteq \nu\left(u^{\prime}\right) \subseteq \nu(w)$ and $f_{i}[\nu(u)] \subseteq\left(a-2^{-n} ; a+\right.$ $\left.2^{-n}\right)$. This proves the first statement.

(2) Let the machine from the above proof search for some $u$ such that additionally $x \in \nu(u)$.

$\square$ (Proposition 6.6)

We define a computable metric space $\mathbf{M}=\left(M, d_{M}, A, \alpha\right)$ as the constructive completion of a computable notated pseudometric space $\mathbf{A}^{\prime}=\left(A^{\prime}, d^{\prime}, \alpha^{\prime}\right)[9$, Definition 8.1.5] which will be constructed now. Let $A^{\prime}:=\operatorname{dom}(\nu)$ and $\alpha^{\prime}(u):=u$ for $u \in A^{\prime}$. Iterating a computable realization of the multi-function $g_{1}$ from Proposition 6.6(1) for every $w \in A^{\prime}$ we can compute a sequence $\left(\left(u_{w k}, a_{w k}\right)\right)_{k \in \mathbb{N}}\left(\right.$ where $\left.\left(u_{w k}, a_{w k}\right) \in A^{\prime} \times \mathbb{Q}\right)$ such that for $k=\langle i, n\rangle$,

$$
\begin{gathered}
\nu\left(u_{w, k+1}\right) \subseteq \nu\left(u_{w k}\right) \subseteq \nu(w), \\
f_{i}\left[\nu\left(u_{w k}\right)\right] \subseteq\left(a_{w k}-2^{-n} ; a_{w k}+2^{-n}\right) .
\end{gathered}
$$

Then for $v, w \in A^{\prime}$ define

$$
d^{\prime}(v, w):=\sup _{k} d\left(\nu\left(u_{v k}\right), \nu\left(u_{w k}\right)\right) .
$$

By (6.5) the sequence $\left(\mathrm{dm} \circ \nu\left(u_{w k}\right)\right)_{k \in \mathbb{N}}$ of diameters is decreasing and by (6.6),

$$
\operatorname{dm}_{i} \circ \nu\left(u_{w\langle i, n\rangle}\right) \leq 2 \cdot 2^{-n} .
$$

Let $x, y \in \nu\left(u_{w\langle n, n\rangle}\right)$. Then for all $j \leq n,\langle j, n\rangle \leq\langle n, n\rangle$, hence $x, y \in \nu\left(u_{w\langle j, n\rangle}\right)$ by (6.5) and therefore, $\left|f_{j}(x)-f_{j}(y)\right| \leq 2 \cdot 2^{-n}$ by (6.8). Since range $\left(f_{j}\right) \subseteq[0 ; 1]$,

$$
\begin{aligned}
d(x, y) & =\sum_{j \in \mathbb{N}} 2^{-j}\left|f_{j}(x)-f_{j}(y)\right| \\
& \leq \sum_{j \leq n} 2^{-j}\left|f_{j}(x)-f_{j}(y)\right|+2^{-n} \\
& \leq \sum_{j \leq n} 2^{-j} \cdot 2 \cdot 2^{-n}+2^{-n} \leq 5 \cdot 2^{-n}
\end{aligned}
$$

and hence,

$$
\operatorname{dm}\left(\nu\left(u_{w\langle n, n\rangle}\right)\right) \leq 5 \cdot 2^{-n}
$$


Since $(\forall k)(\exists n) k \leq\langle n, n\rangle$, the sequence $\left(\mathrm{dm} \circ \nu\left(u_{w k}\right)\right)_{k \in \mathbb{N}}$ converges to 0 .

For $U_{k}:=\nu\left(u_{w_{1} k}\right), V_{k}:=\nu\left(u_{w_{2} k}\right)$ and $W_{k}:=\nu\left(u_{w_{3} k}\right)$,

$$
\begin{aligned}
d\left(U_{k}, W_{k}\right) & \leq d\left(U_{k}, V_{k}\right)+d\left(V_{k}, W_{k}\right)+\operatorname{dm}\left(U_{k}\right)+\operatorname{dm}\left(V_{k}\right)+\operatorname{dm}\left(W_{k}\right) \\
& \leq d^{\prime}\left(w_{1}, w_{2}\right)+d^{\prime}\left(w_{2}, w_{3}\right)+\operatorname{dm}\left(U_{k}\right)+\operatorname{dm}\left(V_{k}\right)+\operatorname{dm}\left(W_{k}\right),
\end{aligned}
$$

hence $d^{\prime}\left(w_{1}, w_{3}\right) \leq d^{\prime}\left(w_{1}, w_{2}\right)+d^{\prime}\left(w_{2}, w_{3}\right)$. Therefore, $d^{\prime}$ is a pseudometric.

We will show that $d^{\prime}$ is computable. Since we have assumed that the base elements of the space $\mathbf{X}$ are not empty, for every $w, k$ there is some $x_{w k} \in \nu\left(u_{w k}\right)$. Although we are not able to compute such points we will use their existence. For $m>n, x_{v\langle i, m\rangle} \in \nu\left(u_{v\langle i, m\rangle}\right) \subseteq$ $\nu\left(u_{v\langle i, n\rangle}\right)$. Then by (6.6), $\left|a_{v\langle i, m\rangle}-a_{v\langle i, n\rangle}\right| \leq 2 \cdot 2^{-n}$. Therefore the sequence $\left(a_{v\langle i, n\rangle}\right)_{n \in \mathbb{N}}$ converges to some $b_{v i} \in \mathbb{R}$ such that $\left|b_{v i}-a_{v\langle i, n\rangle}\right| \leq 2 \cdot 2^{-n}$. The function $(v, i) \mapsto b_{v i}$ is computable. Furthermore, $\left|a_{v\langle i, n\rangle}-a_{w\langle i, n\rangle}\right| \leq\left|a_{v\langle i, n\rangle}-b_{v i}\right|+\left|b_{v i}-b_{w i}\right|+\left|b_{w i}-a_{w\langle i, n\rangle}\right| \leq$ $\left|b_{v i}-b_{w i}\right|+4 \cdot 2^{-n}$ and correspondingly $\left|b_{v i}-b_{w i}\right| \leq\left|a_{v\langle i, n\rangle}-a_{w\langle i, n\rangle}\right|+4 \cdot 2^{-n}$, hence ||$b_{v i}-b_{w i}|-| a_{v\langle i, n\rangle}-a_{w\langle i, n\rangle}|| \leq 4 \cdot 2^{-n}$. By ([6.6) for $k=\langle i, n\rangle,\left|a_{v k}-a_{w k}\right|-2 \cdot 2^{-n} \leq$ $\left|f_{i}\left(x_{v k}\right)-f_{i}\left(x_{w k}\right)\right| \leq\left|a_{v k}-a_{w k}\right|+2 \cdot 2^{-n}$, hence ||$a_{v k}-a_{w k}\left|-d_{i}\left(x_{v k}, x_{w k}\right)\right| \leq 2 \cdot 2^{-n}$. Therefore,

$$
|| b_{v i}-b_{w i}\left|-d_{i}\left(x_{v\langle i, n\rangle}, x_{w\langle i, n\rangle}\right)\right| \leq 6 \cdot 2^{-n} .
$$

Suppose $m \geq\langle i, n\rangle$. Since $x_{v m} \in \nu\left(u_{v m}\right) \subseteq \nu\left(u_{v\langle i, n\rangle}\right)$ and $x_{v\langle i, n\rangle} \in \nu\left(u_{v\langle i, n\rangle}\right)$,

$d_{i}\left(x_{v\langle i, n\rangle}, x_{v m}\right) \leq 2 \cdot 2^{-n}$ by ([6.8) and correspondingly $d_{i}\left(x_{w\langle i, n\rangle}, x_{w m}\right) \leq 2 \cdot 2^{-n}$, hence

$$
\left|d_{i}\left(x_{v m}, x_{w m}\right)-d_{i}\left(x_{v\langle i, n\rangle}, x_{w\langle i, n\rangle}\right)\right| \leq 4 \cdot 2^{-n},
$$

and with (6.10),

$$
|| b_{v i}-b_{w i}\left|-d_{i}\left(x_{v m}, x_{w m}\right)\right| \leq 10 \cdot 2^{-n} .
$$

Then for $N \in \mathbb{N}$ and $m>\langle N+1, N+6\rangle$,

$$
\begin{aligned}
& \left|d\left(x_{v m}, x_{w m}\right)-\sum_{i=0}^{N+1} 2^{-i}\right| b_{v i}-b_{w i}|| \\
= & \left|\sum_{i \in \mathbb{N}} 2^{-i} \cdot d_{i}\left(x_{v m}, x_{w m}\right)-\sum_{i=0}^{N+1} 2^{-i}\right| b_{v i}-b_{w i}|| \\
\leq & \left|\sum_{i=0}^{N+1} 2^{-i} \cdot d_{i}\left(x_{v m}, x_{w m}\right)-\sum_{i=0}^{N+1} 2^{-i}\right| b_{v i}-b_{w i}||+2^{-N-1} \\
= & \left|\sum_{i=0}^{N+1} 2^{-i} \cdot\left(d_{i}\left(x_{v m}, x_{w m}\right)-\left|b_{v i}-b_{w i}\right|\right)\right|+2^{-N-1} \\
\leq & \sum_{i=0}^{N+1} 2^{-i} \cdot\left|d_{i}\left(x_{v m}, x_{w m}\right)-\right| b_{v i}-b_{w i}||+2^{-N-1} \\
\leq & \sum_{i=0}^{N+1} 2^{-i} \cdot 10 \cdot 2^{-N-6}+2^{-N-1} \\
\leq & 20 \cdot 2^{-N-6}+2^{-N-1}<2^{-N} .
\end{aligned}
$$


Since $d\left(\nu\left(u_{v m}\right), \nu\left(u_{w m}\right)\right) \leq d\left(x_{v m}, x_{w m}\right) \leq d\left(\nu\left(u_{v m}\right), \nu\left(u_{w m}\right)\right)+\operatorname{dm}\left(\nu\left(u_{v m}\right)+\operatorname{dm}\left(\nu\left(u_{w m}\right)\right.\right.$,

$$
\lim _{m \rightarrow \infty} d\left(\nu\left(u_{v m}\right), \nu\left(u_{w m}\right)\right)=\lim _{m \rightarrow \infty} d\left(x_{v m}, x_{w m}\right)=d^{\prime}(v, w)
$$

Therefore by the above estimation,

$$
\mid\left[d^{\prime}(v, w)-\sum_{i=0}^{N+1} 2^{-i}\left|b_{v i}-b_{w i}\right| \mid \leq 2^{-N}\right.
$$

for all $N$. Since the function $(v, w, N) \mapsto \sum_{i=0}^{N+1} 2^{-i}\left|b_{v i}-b_{w i}\right|$ is computable, the pseudometric $d^{\prime}$ on the pseudometric space $\mathbf{A}^{\prime}=\left(A^{\prime}, d^{\prime}, \alpha^{\prime}\right)$ is $\left(\alpha^{\prime}, \alpha^{\prime}, \rho\right)$-computable.

Let $\mathbf{M}=\left(M, d_{M}, A, \alpha\right)$ be the constructive completion of the computable notated pseudometric space $\mathbf{A}^{\prime}=\left(A^{\prime}, d^{\prime}, \alpha^{\prime}\right)$, see [9, Definition 8.1.5]. We summarize its definition. Define a set $S$, a function $d_{S}: S \times S \rightarrow \mathbb{R}$ and a binary relation $\sim$ on $S$ as follows:

$$
\begin{gathered}
S:=\left\{\left(w_{0}, w_{1}, \ldots\right) \mid w_{i} \in A^{\prime}, d^{\prime}\left(w_{i}, w_{j}\right) \leq 2^{-i} \text { for } j>i\right\}, \\
d_{S}\left(\left(v_{0}, v_{1}, \ldots\right),\left(w_{0}, w_{1}, \ldots\right)\right):=\lim _{i \rightarrow \infty} d^{\prime}\left(v_{i}, w_{i}\right), \\
\left(v_{0}, v_{1}, \ldots\right) \sim\left(w_{0}, w_{1}, \ldots\right) \Longleftrightarrow d_{S}\left(\left(v_{0}, v_{1}, \ldots\right),\left(w_{0}, w_{1}, \ldots\right)\right)=0 .
\end{gathered}
$$

Then define $M:=S / \sim, d_{M}:=d_{S} / \sim, \alpha(w):=(w, w, w, \ldots) / \sim$ for $w \in \operatorname{dom}(\alpha):=$ $\operatorname{dom}\left(\alpha^{\prime}\right)=\operatorname{dom}(\nu)=A^{\prime}$ and $A:=\operatorname{range}(\alpha)$.

The Cauchy representation $\delta_{C}$ for $\mathbf{M}$ is defined by: $p \in \operatorname{dom}\left(\delta_{C}\right)$ iff there are words $w_{0}, w_{1}, \ldots \in \operatorname{dom}(\alpha)$ such that $p=\iota\left(w_{0}\right) \iota\left(w_{1}\right) \ldots$ and $d^{\prime}\left(w_{i}, w_{j}\right) \leq 2^{-i}$ for $j>i$, and $\delta_{C}(p)=\left(w_{0}, w_{1}, \ldots\right) / \sim$.

We will define a function $f: X \rightarrow M$ and prove that $f$ is well-defined, injective and $\left(\delta, \delta_{M}\right)$-computable and that the partial function $f^{-1}$ is $\left(\delta_{M}, \delta\right)$-computable. For every $w \in \operatorname{dom}(\nu)$ let $\left(\left(u_{w k}, a_{w k}\right)\right)_{k \in \mathbb{N}}$ be the sequence satisfying (6.5, 6.6) for $k=\langle i, n\rangle$ that has been used for defining the pseudometric space $\mathbf{A}^{\prime}$.

Let $\delta(p)=x$. There is a machine $N$ that on input $p \in \Sigma^{\omega}$ first finds some $w \ll p$. Using a computable realization of the multi-function $g_{2}$ from Proposition 6.6(2) from $p$ and $w$ it computes a sequence $\left(\left(v_{p w k}, c_{p w k}\right)\right)_{k \in \mathbb{N}}\left(\right.$ where $\left.\left(v_{p w k}, c_{p w k}\right) \in A^{\prime} \times \mathbb{Q}\right)$ such that for $k=\langle i, n\rangle$,

$$
\begin{gathered}
x \in \nu\left(v_{p w, k+1}\right) \subseteq \nu\left(v_{p w k}\right) \subseteq \nu(w), \\
f_{i}\left[\nu\left(v_{p w k}\right)\right] \subseteq\left(c_{p w k}-2^{-n} ; c_{p w k}+2^{-n}\right) .
\end{gathered}
$$

(compare with (6.5, 6.6) ) and writes the sequence $q:=\iota\left(v_{0}\right) \iota\left(v_{1}\right) \ldots$ where $v_{n}:=v_{p w\langle n+3, n+3\rangle}$. In the same way as above from $(6.5,6.6)$ from $(6.15,6.16)$ we can conclude $\operatorname{dm}\left(\nu\left(v_{p w\langle n, n\rangle}\right)\right) \leq$ $5 \cdot 2^{-n}$. Then

$$
x \in \nu\left(v_{n+1}\right) \subseteq \nu\left(v_{n}\right) \text { and } \operatorname{dm}\left(v_{n}\right)<2^{-n} .
$$

We show $q \in \operatorname{dom}\left(\delta_{C}\right)$. Suppose $i<j$ and let $n \in \mathbb{N}$. Since $\nu\left(v_{j}\right) \subseteq \nu\left(v_{i}\right)$ by (6.5), $\nu\left(u_{v_{i}\langle n, n\rangle}\right) \subseteq \nu\left(v_{i}\right)$ and $\nu\left(u_{v_{j}\langle n, n\rangle}\right) \subseteq \nu\left(v_{i}\right)$, hence $d\left(\nu\left(u_{v_{i}\langle n, n\rangle}\right), \nu\left(u_{v_{j}\langle n, n\rangle}\right)\right) \leq \operatorname{dm} \circ \nu\left(v_{i}\right)$. Therefore by (6.7) and (6.17),

$$
d^{\prime}\left(v_{i}, v_{j}\right)=\sup _{n} d\left(\nu\left(u_{v_{i}\langle n, n\rangle}\right), \nu\left(u_{v_{j}\langle n, n\rangle}\right)\right) \leq \operatorname{dm} \circ \nu\left(v_{i}\right) \leq 2^{-i} .
$$

Therefore, $q \in \operatorname{dom}\left(\delta_{C}\right)$.

Let $\delta(p)=x, \delta\left(p^{\prime}\right)=x^{\prime}, f_{N}(p)=q=\iota\left(v_{0}\right) \iota\left(v_{1}\right) \ldots$ and $f_{N}\left(p^{\prime}\right)=q^{\prime}=\iota\left(v_{0}^{\prime}\right) \iota\left(v_{1}^{\prime}\right) \ldots$ By the definition of $d_{M}, d_{M}\left(\delta_{C}(q), \delta_{C}\left(q^{\prime}\right)\right)=d_{S}\left(\left(v_{0}, v_{1}, \ldots\right),\left(v_{0}^{\prime}, v_{1}^{\prime}, \ldots\right)\right)=\lim _{i \rightarrow \infty} d^{\prime}\left(v_{i}, v_{i}^{\prime}\right)$. 
For all $i \in \mathbb{N}, x \in \nu\left(v_{i}\right)$ and $x^{\prime} \in \nu\left(v_{i}^{\prime}\right)$ by (6.17) and for all $n \in \mathbb{N}, \nu\left(u_{v_{i}\langle n, n\rangle}\right) \subseteq \nu\left(v_{i}\right)$ and $\nu\left(u_{v_{i}^{\prime}\langle n, n\rangle}\right) \subseteq \nu\left(v_{i}^{\prime}\right)$ by (6.5). For $y \in \nu\left(v_{i}\right)$ and $y^{\prime} \in \nu\left(v_{i}^{\prime}\right)$ by (6.17), $\left|d\left(x, x^{\prime}\right)-d\left(y, y^{\prime}\right)\right| \leq$ $2 \cdot 2^{-i}$. Therefore, $\left|d\left(x, x^{\prime}\right)-d\left(\nu\left(u_{v_{i}\langle n, n\rangle}\right), \nu\left(u_{v_{i}^{\prime}\langle n, n\rangle}\right)\right)\right| \leq 2 \cdot 2^{-i}$. Since by (6.7) $d^{\prime}\left(v_{i}, v_{i}^{\prime}\right)=$ $\lim _{n \rightarrow \infty} d\left(\nu\left(u_{v_{i}\langle n, n\rangle}\right), \nu\left(u_{v_{i}^{\prime}\langle n, n\rangle}\right)\right),\left|d\left(x, x^{\prime}\right)-d^{\prime}\left(v_{i}, v_{i}^{\prime}\right)\right| \leq 2 \cdot 2^{-i}$. Then by (6.13),

$$
d\left(x, x^{\prime}\right)=d_{S}\left(\left(v_{0}, v_{1}, \ldots\right),\left(v_{0}^{\prime}, v_{1}^{\prime}, \ldots\right)\right)=d_{M}\left(\delta_{C}(q), \delta_{C}\left(q^{\prime}\right)\right) .
$$

If $\delta(p)=\delta\left(p^{\prime}\right)$ then $\delta_{C} \circ f_{N}(p)=\delta_{C} \circ f_{N}\left(p^{\prime}\right)$, hence $f_{N}$ realizes a single-valued function $f: X \rightarrow M$. By (6.18),

$$
d\left(x, x^{\prime}\right)=d_{M}\left(f(x), f\left(x^{\prime}\right)\right),
$$

therefore, $f$ is a $\left(\delta, \delta_{C}\right)$-computable isometric function.

Finally, we show that $f^{-1}$ is $\left(\delta_{C}, \delta\right)$-computable. Suppose $f(x)=y=\delta_{C}(q) \in \operatorname{range}(f)$ with $q=\left(\iota\left(w_{0}\right) \iota\left(w_{1}\right) \ldots\right)$. Notice that not necessarily $\nu\left(w_{n+1}\right) \subseteq \nu\left(w_{n}\right)$. There is some $p^{\prime}$ such that $x=\delta\left(p^{\prime}\right)$ and, by the definition of $f, y=f(x)=\delta_{C} \circ f_{N}\left(p^{\prime}\right)$. Then there are words $w_{n}^{\prime}$ such that $q^{\prime}:=f_{N}\left(p^{\prime}\right)=\left(\iota\left(w_{0}^{\prime}\right) \iota\left(w_{1}^{\prime}\right) \ldots\right)$ and $\delta_{C}\left(q^{\prime}\right)=y$. By (6.15, 6.16), for these words, $x \in \nu\left(w_{n+1}^{\prime}\right) \subseteq \nu\left(w_{n}^{\prime}\right), \operatorname{dm} \circ \nu\left(w_{n}^{\prime}\right)<2^{-n}$ and hence $\lim _{n \rightarrow \infty} d^{\prime}\left(w_{n}, w_{n}^{\prime}\right)=0$. Since $\delta_{C}(q)=y=\delta_{C}\left(q^{\prime}\right)$,

$$
d^{\prime}\left(w_{n}, w_{n}^{\prime}\right) \leq 2 \cdot 2^{-n} \text { for all } n .
$$

(Still, for every $w \in \operatorname{dom}(\nu)$ let $\left(\left(u_{w k}, a_{w k}\right)\right)_{k \in \mathbb{N}}$ be the sequence satisfying (6.5, 6.6) for $k=\langle i, n\rangle$ that has been used for defining the pseudometric space $\mathbf{A}^{\prime}$.) Since $x \in \nu\left(w_{n}^{\prime}\right)$ and $\nu\left(u_{w_{n}^{\prime}\langle n, n\rangle}\right) \subseteq \nu\left(w_{n}^{\prime}\right), d\left(x, \nu\left(u_{w_{n}^{\prime}\langle n, n\rangle}\right)\right) \leq \operatorname{dm}\left(w_{n}^{\prime}\right) \leq 2^{-n}$.

Suppose $z \in \nu\left(u_{w_{n}\langle n, n\rangle}\right)$. Then $d\left(\nu\left(u_{w_{n}\langle n, n\rangle}\right), z\right)=0$, hence by (6.4, 6.9, 6.20)

$$
\begin{aligned}
d(x, z) \leq & d\left(x, \nu\left(u_{w_{n}^{\prime}\langle n, n\rangle}\right)\right)+d\left(\nu\left(u_{w_{n}^{\prime}\langle n, n\rangle}\right), \nu\left(u_{w_{n}\langle n, n\rangle}\right)\right) \\
& +d\left(\nu\left(u_{w_{n}\langle n, n\rangle}\right), z\right)+10 \cdot 2^{-n} \\
\leq & 2^{-n}+d^{\prime}\left(w_{n}^{\prime}, w_{n}\right)+10 \cdot 2^{-n} \\
\leq & 13 \cdot 2^{-n} .
\end{aligned}
$$

Let $\left(\left(u_{i}, v_{i}\right)\right)_{i \in \mathbb{N}}$ be the computable numbering of the relation $R$ from (3.4) for defining the functions $f_{i}$ in the proof of Theorem 6.3. We prove that $x \in \nu(v)$ iff there are numbers $i, n \in \mathbb{N}$ such that

$$
n \geq i+4, \quad v=v_{i}, \quad \text { and } \quad \nu\left(u_{i}\right) \cap \nu\left(u_{w_{n}\langle n, n\rangle}\right) \neq \emptyset .
$$

Suppose, $x \in \nu(v)$. There is some $i$ such that $v=v_{i}$ and $x \in \nu\left(u_{i}\right) \subseteq \nu\left(v_{i}\right)$. There is some $j$ such that $x \in B\left(x, 2^{-j}\right) \subseteq \nu\left(u_{i}\right)$. Let $n:=\max (j+4, i+4)$. Then for all $z \in \nu\left(u_{w_{n}\langle n, n\rangle}\right), \quad d(x, z) \leq 13 \cdot 2^{-n}<2^{-j}$. We conclude $\nu\left(u_{w_{n}\langle n, n\rangle}\right) \subseteq B\left(x, 2^{-j}\right) \subseteq \nu\left(u_{i}\right)$, hence $\nu\left(u_{i}\right) \cap \nu\left(u_{w_{n}\langle n, n\rangle}\right)=\nu\left(u_{w_{n}\langle n, n\rangle}\right) \neq \emptyset$ (since $(\forall u) \nu(u) \neq \emptyset$ by assumption).

On the other hand, suppose (6.21) holds for some $i, n \in \mathbb{N}$. There is some $z \in \nu\left(u_{i}\right) \cap$ $\nu\left(u_{w_{n}\langle n, n\rangle}\right)$. Then $d(x, z) \leq 13 \cdot 2^{-n}$ as shown above. Therefore by $n \geq i+4$,

$$
\left|f_{i}(x)-f_{i}(z)\right| \leq 2^{i} \cdot d(x, z) \leq 2^{i} \cdot 13 \cdot 2^{-n}<1 .
$$

Since $z \in \nu\left(u_{i}\right), f_{i}(z)=0$. Therefore, $f_{i}(x)<1$ hence $x \in \nu\left(v_{i}\right)=\nu(v)$ (see (6.1]6.2[6.3) $)$.

Since $(w, n) \mapsto u_{w,\langle n, n\rangle}$ is computable and $\nu(u) \cap \nu(v) \neq \emptyset$ is r.e., by (6.21) from $q=\iota\left(w_{0}\right) \iota\left(w_{1}\right) \ldots$ we can compute a list of all $v$ such that $x \in \nu(v)$. Therefore, $f^{-1}$ is $\left(\delta_{C}, \delta\right)$-computable. 
We mention that by (6.19) the embedding $f$ is an isometric function from the metric space $(X, d)$ into $\left(M, d_{M}\right)$, where $d$ is the metrization of the original $T_{3}$-space $\mathbf{X}$ constructed in the proof of Theorem 6.3. Let NE abbreviate " $U \neq \emptyset$ is $\nu$-r.e.". The condition in the embedding theorem 6.5 is $\left(\mathrm{CT}_{3}+\mathrm{NE}\right)$. By Theorem 4.4 this implies $\mathrm{SCT}_{3}$. We do not know whether $\mathrm{SCT}_{3}$ or $\mathrm{STy}$ are sufficient to prove the embedding theorem.

\section{Separation on Product Spaces}

For a computable topological space $\mathbf{X}=(X, \tau, \beta, \nu)$ and $B \subseteq X$ the subspace $\mathbf{X}_{B}=$ $\left(B, \tau_{B}, \beta_{B}, \nu_{B}\right)$ of $\mathbf{X}$ to $B$ is the computable topological space defined by $\operatorname{dom}\left(\nu_{B}\right):=$ $\operatorname{dom}(\nu), \nu_{B}(w):=\nu(w) \cap B[13$, Section 8]. The separation axioms from Definition [3.2 are invariant under restriction to subspaces.

Theorem 7.1. If a computable topological space satisfies some separation axiom from Definition 3.2 then each subspace satisfies this axiom.

Proof: Straightforward.

The product of two $T_{i}$-spaces is a $T_{i}$-space for $i=0,1,2,3$. This is no longer true for some of the computable separation axioms. The product $\mathbf{X}_{1} \times \mathbf{X}_{2}=\overline{\mathbf{X}}=\left(X_{1} \times X_{2}, \bar{\tau}, \bar{\beta}, \bar{\nu}\right)$ of two computable topological spaces $\mathbf{X}_{1}=\left(X_{1}, \tau_{1}, \beta_{1}, \nu_{1}\right)$ and $\mathbf{X}_{2}=\left(X_{2}, \tau_{2}, \beta_{2}, \nu_{2}\right)$, defined by $\bar{\nu}\left\langle u_{1}, u_{2}\right\rangle=\nu_{1}\left(u_{1}\right) \times \nu_{2}\left(u_{2}\right)$, is again a computable topological space [13, Section 8]. For the next theorem see Figure 1 .

Theorem 7.2.

The $S C T_{2^{-}}, W C T_{3^{-}}, C T_{3^{-}}, C T y$ - and $S C T_{3}$-spaces are closed under finite products.

We consider computability w.r.t. $\nu_{i}, \delta_{i}, \psi_{i}^{-}, \bar{\nu}, \bar{\delta}$ and $\bar{\psi}^{-}$.

Proof: Suppose, $\mathbf{X}_{1}$ and $\mathbf{X}_{2}$ are $S C T_{2}$. By [12, Theorem 7], $x_{i} \neq y_{i}$ is $\left(\delta_{i}, \delta_{i}\right)$-r.e. for $i=1,2$, hence $\left(x_{1}, x_{2}\right) \neq\left(y_{1}, y_{2}\right)$ is $\left(\left[\delta_{1}, \delta_{2}\right],\left[\delta_{1}, \delta_{2}\right]\right)$-r.e., hence again by Theorem 6.3, $\mathbf{X}_{1} \times \mathbf{X}_{2}$ is $S C T_{2}$.

Suppose, $\mathbf{X}_{1}$ and $\mathbf{X}_{2}$ are $W C T_{3}$. Let $\left(x_{1}, x_{2}\right) \in W_{1} \times W_{2}$. From $x_{i}$ and $W_{i}$ we can find $U_{i} \in \beta_{i}$ such that $x_{i} \in U_{i} \subseteq \bar{U}_{i} \subseteq W_{i}$ (for $\left.i=1,2\right)$. Then $\left(x_{1}, x_{2}\right) \in U_{1} \times U_{2} \subseteq \overline{U_{1} \times U_{2}}=$ $\bar{U}_{1} \times \bar{U}_{2} \subseteq W_{1} \times W_{2}$.

Suppose, $\mathbf{X}_{1}$ and $\mathbf{X}_{2}$ are $C T_{3}^{\prime}$. Suppose $\left(x_{1}, x_{2}\right) \in\left(W_{1}, W_{2}\right) \in \beta_{1} \times \beta_{2}$. From $\left(\left(x_{1}, x_{2}\right),\left(W_{1}, W_{2}\right)\right)$ we can compute $x_{1}, x_{2}, W_{1}$ and $W_{2}$. Using $t_{3}^{\prime}$ for $\mathbf{X}_{\mathbf{1}}$ and $\mathbf{X}_{\mathbf{2}}$ we can compute $\left(U_{i}, B_{i}\right)$ such that $U_{i} \in \beta_{i}, B_{i} \subseteq X_{i}$ is closed and $x_{i} \in U_{i} \subseteq B_{i} \subseteq W_{i}(i=1,2)$. Observe that $\left(x_{1}, x_{2}\right) \in U_{1} \times U_{2} \subseteq B_{1} \times B_{2} \subseteq W_{1} \times W_{2}$. Form $\left(U_{1}, B_{1}\right)$ and $\left(U_{2}, B_{2}\right)$ we can compute $\left(\left(u_{1}, u_{2}\right),\left(B_{1}, B_{2}\right)\right)$.

Suppose $\mathbf{X}_{1}$ and $\mathbf{X}_{2}$ are $C T y^{\prime}$. We show that $\mathbf{X}_{1} \times \mathbf{X}_{2}$ is $C T y^{\prime}$. From $\left(x_{1}, x_{2}\right) \in W_{1} \times W_{2}$ where $W_{1} \in \beta_{1}$ and $W_{2} \in \beta_{2}$, we can compute $x_{1}$ and $W_{1}$, where $x_{1} \in W_{1}$. By CTy' for $\mathbf{X}_{1}$, from these data we can compute some $U_{1} \in \beta_{1}$ and a function $f_{1}: X_{1} \rightarrow \mathbb{R}$ such that $x_{1} \in U_{1} \subseteq W_{1}$ and $f_{1}$ is 0 inside $U_{1}$ and 1 outside $W_{1}$. Correspondingly, we can compute $U_{2}$ and $f_{2}$ such that $x_{2} \in U_{2} \subseteq W_{2}$ and $f_{2}$ is 0 inside $U_{2}$ and 1 outside $W_{2}$. From $U_{1}, U_{2}$ we can compute $\bar{U}:=U_{1} \times U_{2}$ and $\bar{f}$ such that $\bar{f}\left(y_{1}, y_{2}\right)=\max \left(f_{1}\left(y_{1}\right), f_{2}\left(y_{2}\right)\right)$. Then $\left(x_{1}, x_{2}\right) \in U_{1} \times U_{2} \subseteq W_{1} \times W_{2}$, and and $\bar{f}$ is 0 inside $U_{1} \times U_{2}$ and 1 outside $W_{1} \times W_{2}$.

For $\mathbf{X}_{\mathbf{i}}(i=1,2)$ let $R_{i}$ be the r.e. set and let $r_{i}$ be the computable function for $S C T_{3}$ from Definition [3.2, By [13, Lemma 27] there is a computable function $h$ such that 


$$
\begin{aligned}
& \psi_{1}^{-}\left(p_{1}\right) \times \psi_{2}^{-}\left(p_{2}\right)=\bar{\psi}^{-} \circ h\left(p_{1}, p_{2}\right) \text {. Let } \\
& \bar{R}:=\left\{\left(\left\langle u_{1}, u_{2}\right\rangle,\left\langle w_{1}, w_{2}\right\rangle\right) \mid\left(u_{1}, w_{1}\right) \in R_{1} \wedge\left(u_{2}, w_{2}\right) \in R_{2}\right\}, \\
& \quad \bar{r}\left(\left\langle u_{1}, u_{2}\right\rangle,\left\langle w_{1}, w_{2}\right\rangle\right):=h\left(r_{1}\left(u_{1}, w_{1}\right), r_{2}\left(u_{2}, w_{2}\right)\right) .
\end{aligned}
$$

A straightforward calculation shows that $\bar{R}$ is the r.e. set and $\bar{r}$ be the computable function for $S C T_{3}$ from the definition for the product $\mathbf{X}_{1} \times \mathbf{X}_{2}$.

The $C T_{2}$-spaces are not closed under product [12, Theorem 15]. Presumably, the $C T_{4^{-}}$ spaces, and hence the $C U r$-spaces, are not closed under product.

\section{FinAl REMARKS AND THANKS}

The list of axioms of computable separation in Definition 3.2 is not exhaustive, there may be other ones. Applications must show which of these axioms are the most natural and useful ones. Many questions about the logical relation between the given axioms have not been answered.

I thank the unknown referees for very careful reading and for giving a number of useful remarks.

\section{REFERENCES}

[1] Andrej Bauer, Peter Hertling, and Ker-I Ko, editors. Sixth International Conference on Computability and Complexity in Analysis, CCA 2009, August 18-22, 2009, Ljubljana, Slovenia, volume 11 of OASICS. Schloss Dagstuhl - Leibniz-Zentrum fuer Informatik, Germany, 2009.

[2] Vasco Brattka, Peter Hertling, and Klaus Weihrauch. A tutorial on computable analysis. In S. Barry Cooper, Benedikt Löwe, and Andrea Sorbi, editors, New Computational Paradigms: Changing Conceptions of What is Computable, pages 425-491. Springer, New York, 2008.

[3] Ryszard Engelking. General Topology, volume 6 of Sigma series in pure mathematics. Heldermann, Berlin, 1989.

[4] Tanja Grubba, Matthias Schröder, and Klaus Weihrauch. Computable metrization. Mathematical Logic Quarterly, 53(4-5):381-395, 2007.

[5] Tanja Grubba, Klaus Weihrauch, and Yatao Xu. Effectivity on continuous functions in topological spaces. In Ruth Dillhage, Tanja Grubba, Andrea Sorbi, Klaus Weihrauch, and Ning Zhong, editors, CCA 2007, Fourth International Conference on Computability and Complexity in Analysis, volume 338 of Informatik Berichte, pages 137-154. FernUniversität in Hagen, June 2007. CCA 2007, Siena, Italy, June 16-18, 2007.

[6] Christoph Kreitz and Klaus Weihrauch. Theory of representations. Theoretical Computer Science, 38:35$53,1985$.

[7] Matthias Schröder. Effective metrization of regular spaces. In Ker-I Ko, Anil Nerode, Marian B. PourEl, Klaus Weihrauch, and Jiř́ Wiedermann, editors, Computability and Complexity in Analysis, volume 235 of Informatik Berichte, pages 63-80. FernUniversität Hagen, August 1998. CCA Workshop, Brno, Czech Republic, August, 1998.

[8] Klaus Weihrauch. Computability on computable metric spaces. Theoretical Computer Science, 113:191210, 1993. Fundamental Study.

[9] Klaus Weihrauch. Computable Analysis. Springer, Berlin, 2000.

[10] Klaus Weihrauch. The computable multi-functions on multi-represented sets are closed under programming. Journal of Universal Computer Science, 14(6):801-844, 2008.

[11] Klaus Weihrauch. Computable separation in topology, from $T_{0}$ to $T_{3}$. In $C C A, 2009$.

[12] Klaus Weihrauch. Computable separation from $T_{0}$ to $T_{2}$. Journal of Universal Computer Science, 16(18):2733-2753, 2010.

[13] Klaus Weihrauch and Tanja Grubba. Elementary computable topology. Journal of Universal Computer Science, 15(6):1381-1422, 2009. 
[14] Yatao Xu and Tanja Grubba. On computably locally compact Hausdorff spaces. Mathematical Structures in Computer Science, 19:101-117, 2009.

\section{Appendix A (Proof of Theorem 4.1(4))}

$\mathbf{S C T}_{\mathbf{3}} \Longrightarrow \mathbf{C T}_{\mathbf{4}}$ : Let $A, B$ be disjoint closed sets. Suppose there are sequences of open sets $V_{i}, W_{i}$ and of closed sets $S_{i}, T_{i}(i=0,1, \ldots)$ such that

$$
\begin{gathered}
V_{i} \subseteq S_{i}, \quad W_{i} \subseteq T_{i}, \\
A \subseteq \bigcup_{j \in \mathbb{N}} W_{j}, \quad B \cap T_{i}=\emptyset, \\
B \subseteq \bigcup_{j} V_{j \in \mathbb{N}}, \quad A \cap S_{i}=\emptyset .
\end{gathered}
$$

For $i \in \mathbb{N}$ let

$$
G_{i}:=W_{i} \backslash \bigcup_{j \leq i} S_{i}, \quad H_{i}:=V_{i} \backslash \bigcup_{j \leq i} T_{i}
$$

By (8.28.3),

$$
A \subseteq O_{A}:=\bigcup_{i} G_{i}, \quad B \subseteq O_{B}:=\bigcup_{i} H_{i}
$$

The sets $O_{A}$ and $O_{B}$ are open. By (8.4) for $j \leq i, \quad G_{i} \cap S_{j}=\emptyset$ and so $G_{i} \cap V_{j}=\emptyset$. Therefore, $G_{i} \cap H_{j}=\emptyset$ for $j \leq i$. Similarly, $H_{i} \cap G_{j}=\emptyset$ for $j \leq i$. Therefore, $G_{i} \cap H_{j}=\emptyset$ for $i, j \in \mathbb{N}$ and so $O_{A} \cap O_{B}=\emptyset$.

It remains to show that sets $O_{A}$ and $O_{B}$ can be computed from $A$ and $B$. Assume $\psi^{-}(p)=A$ and $\psi^{-}(q)=B$. From $p$ and $q$ sequences of pairs $\left(v_{i}, v_{i}^{p}\right)$ and $\left(w_{i}, v_{i}^{q}\right)$ of words can be computed such that

$$
\begin{aligned}
& \{(u, v) \in R \mid v \ll p\}=\left\{\left(v_{0}, v_{0}^{p}\right),\left(v_{1}, v_{1}^{p}\right), \ldots\right\} \\
& \{(u, v) \in R \mid v \ll q\}=\left\{\left(w_{0}, v_{0}^{q}\right),\left(w_{1}, v_{1}^{q}\right), \ldots\right\},
\end{aligned}
$$

where $R$ is the r.e. set from $\left(\mathrm{SCT}_{3}\right)$. For $i \in \mathbb{N}$ let

$$
\begin{array}{rlrl}
V_{i}:=\nu\left(v_{i}\right), & & S_{i}:=\psi^{-} \circ r\left(v_{i}, v_{i}^{p}\right), \\
W_{i}:=\nu\left(w_{i}\right), & T_{i}:=\psi^{-} \circ r\left(w_{i}, v_{i}^{q}\right) .
\end{array}
$$

Then (8.188.28.3) hold true. By [13, Theorem 11] finite intersection and countable union of open sets can be computed, therefore, from the $V_{i}, S_{i}, W_{i}$ and $T_{i}$ the open sets sets $O_{A}$ and $O_{B}$ defined in (8.4]8.5) can be computed, for which $A \subseteq O_{A}, B \subseteq O_{B}$ and $O_{A} \cap O_{B}=\emptyset$. Therefore, the multi-function $t_{4}$ is $\left(\psi^{-}, \psi^{-},[\theta, \theta]\right)$-computable.

\section{Appendic B, Proof of Theorem 4.1,(6)}

$\mathbf{C U r} \Longrightarrow \mathbf{C T}_{4}:$ See the proof of Theorem 4.1 .

$\mathbf{C T}_{\mathbf{4}} \Longrightarrow \mathbf{C U r}$ : We effectivize the classical proof from [3]. Suppose the space is $C T_{4}$. Then the multi-function

$$
t:(D, U) \mapsto(V, C) \text { for open } U, V \text { and closed } C, D \text { such that } D \subseteq V \subseteq C \subseteq U
$$


is computable. (Find $(V, W) \in t_{4}\left(D, U^{c}\right)$ and let $C:=W^{c}$.) From closed disjoint sets $A, B$ we compute a family $\left(V_{a}, C_{a}\right), a \in \mathbb{Q} \cap[0 ; 1]$, of pairs of sets such that

$$
\begin{aligned}
& V_{a} \text { is open, } C_{a} \text { is closed, } V_{a} \subseteq C_{a}, \\
& C_{a} \subseteq V_{b} \text { if } a<b, \\
& A \subseteq V_{0}, \quad C_{1} \subseteq B^{c} .
\end{aligned}
$$

For this purpose let $i \mapsto r_{i}$ be a canonical bijective numbering of the rational numbers from the interval $[0 ; 1]$ such that $r_{0}=0$ and $r_{1}=1$. Define recursively

$$
\left(V_{0}, C_{0}\right) \in t\left(A, B^{c}\right), \quad\left(V_{1}, C_{1}\right) \in t\left(C_{0}, B^{c}\right), \quad\left(V_{k}, C_{k}\right) \in t\left(C_{l}, V_{m}\right)
$$

such that $r_{l}$ is the maximum of the numbers in $\left\{r_{0}, \ldots, r_{k-1}\right\}$ which are less than $r_{k}$ and $r_{m}$ is the minimum of the numbers in $\left\{r_{0}, \ldots, r_{k-1}\right\}$ which are greater than $r_{k}$. The properties (8.7/8.8 8.9 ) can be verified easily. We define two real valued functions $f_{<}$and $f_{>}$on $X$ as follows:

$$
\begin{aligned}
& f_{<}(x):=\sup \left(\left\{a \mid x \notin C_{a}\right\} \cup\{0\}\right), \\
& f_{>}(x):=\inf \left(\left\{a \mid x \in V_{a}\right\} \cup\{1\}\right) .
\end{aligned}
$$

If $x \in V_{a}$ and $b>a$ then $x \in C_{b}$, hence $b \leq a$ if $x \in V_{a}$ and $x \notin C_{b}$. Therefore, $f_{<}(x) \leq f_{>}(x)$. Suppose, $f_{<}(x)>f_{>}(x)$ for some $x$. Then there is some $c \in \mathbb{Q}$ such that $f_{<}(x)>c>f_{>}(x)$. Moreover, there are some $b>c$ such that $x \notin C_{b}$ and some $a<c$ such that $x \in V_{a}$. But $a<b$ and $x \in V_{a}$ implies $x \in C_{b}$. Contradiction. Therefore, $f:=f_{<}=f_{>}$. The function $f$ has value 0 on $A$ and value 1 on $B$ and is continuous [3].

We show that $f$ can be computed from $A$ and $B$. Since the function $t$ in (8.6) is computable, the function $(A, B) \mapsto\left(V_{r_{i}}, C_{r_{i}}\right)_{i}$ is $\left(\psi, \psi,[\theta, \psi]^{\omega}\right)$-computable by (8.10). By [13, Theorem 13.2], $x \in U$ for open $U$ is $(\rho, \theta)$-r.e. and $x \notin C$ for closed $C$ is $(\rho, \psi)$-r.e. Therefore, from $\left(V_{r_{i}}, C_{r_{i}}\right)_{i}$ and $x$ by (8.11) we can list all $a \in \mathbb{Q}$ such that $a<f(x)$ and by (8.12) we can list all $a \in \mathbb{Q}$ such that $a>f(x)$. Therefore, the function $\left(\left(V_{r_{i}}, C_{r_{i}}\right)_{i}, x\right) \mapsto f(x)$ is $\left.\left([\theta, \psi]^{\omega}\right), \delta, \rho\right)$-computable, hence by type conversion [9, Lemma 3.3.15] $\left(V_{r_{i}}, C_{r_{i}}\right)_{i} \mapsto f$ is $\left.\left([\theta, \psi]^{\omega}\right),[\delta \rightarrow \rho]\right)$-computable. Therefore, the space is $C U r$. 